\title{
Joint Beamforming and Broadcasting in Massive MIMO
}

\author{
Erik G. Larsson and H. Vincent Poor
}

\begin{abstract}
The downlink of a massive MIMO system is considered for the case in which the base station must concurrently serve two categories of terminals: one group to which imperfect instantaneous channel state information (CSI) is available, and one group to which no CSI is available. Motivating applications include broadcasting of public channels and control information in wireless networks.

A new technique is developed and analyzed: joint beamforming and broadcasting (JBB), by which the base station beamforms to the group of terminals to which CSI is available, and broadcasts to the other group of terminals, to which no CSI is available. The broadcast information does not interfere with the beamforming as it is placed in the nullspace of the channel matrix collectively seen by the terminals targeted by the beamforming. JBB is compared to orthogonal access (OA), by which the base station partitions the time-frequency resources into two disjunct parts, one for each group of terminals.
\end{abstract}

It is shown that $\mathrm{JBB}$ can substantially outperform $\mathrm{OA}$ in terms of required total radiated power for given rate targets.

\section{INTRODUCTION}

Massive MIMO [1] is a leading technology candidate for 5G wireless access. The main concept is that hundreds of base station antennas act phase-coherently together and serve tens of terminals in the same time-frequency resource. Different base stations, however, do not cooperate. A fundamental assumption in massive MIMO is that the base station antenna array can acquire instantaneous channel state information (CSI) to the terminals, so that closed-loop beamforming can be applied. This is possible by operating in time-division duplex (TDD) mode, with the base station acquiring CSI from uplink pilots, and relying on reciprocity of the propagation channel.

In wireless networks, the base station will also need to broadcas 11 information to terminals to which it has no CSI. Practical examples of when broadcasting is desired in cellular

E. G. Larsson is with the Dept. of Electrical Engineering (ISY), Linköping University, Linköping, Sweden. H. V. Poor is with the Dept. of Electrical Engineering, Princeton University, Princeton, NJ, USA. Parts of this work were performed when the first author was a visiting fellow at Princeton University.

This work was supported in part by the Swedish Research Council (VR), ELLIIT, and the U.S. National Science Foundation under Grants CNS1456793 and ECCS-1343210.

(C) 2016 IEEE. Personal use of this material is permitted. Permission from IEEE must be obtained for all other uses, in any current or future media, including reprinting/republishing this material for advertising or promotional purposes, creating new collective works, for resale or redistribution to servers or lists, or reuse of any copyrighted component of this work in other works.

This paper will appear in the IEEE Transactions on Wireless Communications, 2016, DOI: 10.1109/TWC.2016.2515598.

${ }^{1}$ The word "broadcast" here means transmitting common data intended to an unknown number of terminals, and must not be confused with the "broadcast channel" in information theory. systems include: delivery of broadcast content [2]; evolved multimedia broadcast/multicast services [3]; the transmission of public "beacon" channels; and the transmission of userspecific control messages intended to "wake up" a particular terminal and instruct it to send uplink pilots.

When CSI is unavailable at the base station, beamforming is impossible and the only way of benefitting from multiple antennas is to use space-time coding, which does not offer multiplexing or array gains. Throughout, we call the terminals to which beamforming is performed (using imperfect, instantaneous CSI) "B-terminals", and all other terminals in the cell (for which no CSI is available) "O-terminals". In general, there is an arbitrary number of O-terminals in the cell.

There are two main ways of accommodating the broadcasting functionality:

1) A fraction $\epsilon$ of the available time-frequency resources can be set aside for the broadcasting to the O-terminals. The remaining fraction, $1-\epsilon$, of the resources, are then used for beamforming to the B-terminals. This approach is termed orthogonal access (OA) here.

2) As proposed in preliminary form in [4] and further developed here, the base station may concurrently beamform coherently to the B-terminals and broadcast to the O-terminals. This is made possible by placing the signals aimed at the O-terminals in the nullspace of the channel matrix of the B-terminals. This scheme, called joint beamforming and broadcasting (JBB) here, is in turn possible owing to the surplus of spatial degrees of freedom in massive MIMO.

This paper analyzes and compares $\mathrm{OA}$ and JBB in terms of required radiated power for given rate targets, taking into account the effects of channel estimation errors and power control.

\section{A. Related Work}

The need for efficient solutions to broadcasting of public information in wireless networks using massive MIMO technology has been recognized before by us [5] and others [6]. However, no known papers address the specific problem at hand. Remotely related, reference [7] proposed schemes for multicasting to a known set of terminals for which imperfect instantaneous CSI is available. Multicasting with per-antenna power constraints was introduced in [8], and specifically for large antenna arrays in [9]. Reference [10] considered combined broadcast/multicast transmission of common and private symbols, which is a different problem. 
JBB exploits the surplus of spatial degrees of freedom in massive MIMO systems. In this context, it is worth pointing out that there are also other possible uses of these excess degrees of freedom: notably, to achieve secrecy by transmitting artificial noise into the channel nullspace [11], [12]; to produce per-antenna waveforms with reduced peak-to-average ratios [13]-[15]; and to suppress out-of-cell interference [16].

Rigorous capacity bounds for massive MIMO beamforming performance are available in the literature: [17] for the downlink, and [18] for the uplink, most notably. Some of our analysis uses techniques and results from these references. However, none of these references dealt with the problem of joint beamforming and broadcasting.

\section{Preliminaries: Massive MiMO Beamforming}

We consider a single cell comprising a base station with an array of $M$ antennas, that serves $K$ single-antenna Bterminals; $K<M$. Let $\boldsymbol{g}_{k}$ be an $M$-vector that represents the channel response, from the array to the $k$ th B-terminal, in a given coherence interval. "Coherence interval" here means the time-frequency space over which the channel is substantially static. We denote by $\tau_{c}$ the length (in samples) of a coherence interval.

In the downlink, at time $t$ ("time" here means sample index in a given coherence interval), the base station transmits the $M$-vector

$$
\boldsymbol{x}(t)=\sqrt{\rho_{b}} \cdot \sum_{k=1}^{K} \boldsymbol{v}_{k} s_{k}(t),
$$

where $\left\{\boldsymbol{v}_{k}\right\}$ are beamforming vectors associated with the $K$ terminals, $\left\{s_{k}(t)\right\}$ are symbols aimed at the $K$ terminals at time instant $t$, and $\rho_{b}$ is the downlink power. The symbols $\left\{s_{k}(t)\right\}$ are assumed to have zero means and unit variances. The beamforming vectors $\left\{\boldsymbol{v}_{k}\right\}$ are functions of estimates of the channel responses $\left\{\boldsymbol{g}_{k}\right\}$, and normalized such that ${ }^{2}$

$$
\mathrm{E}\left[\left\|\sum_{k=1}^{K} \boldsymbol{v}_{k} s_{k}(t)\right\|^{2}\right]=\mathrm{E}\left[\sum_{k=1}^{K}\left\|\boldsymbol{v}_{k}\right\|^{2}\right]=1 .
$$

Operationally the beamforming in (1) makes sure that power emitted by the base station array is focused onto the terminals.

The $k$ th B-terminal sees an effective scalar channel with gain $\boldsymbol{g}_{k}^{H} \boldsymbol{v}_{k}$. In this paper, we assume that no pilots are transmitted on the downlink, and that the B-terminal detects the downlink data coherently by assuming that the gain $\boldsymbol{g}_{k}^{H} \boldsymbol{v}_{k}$ is equal to its expected value $E\left[\boldsymbol{g}_{k}^{H} \boldsymbol{v}_{k}\right]$. This assumption can be justified thanks to channel hardening: by the law of large numbers, $\boldsymbol{g}_{k}^{H} \boldsymbol{v}_{k} \approx \mathrm{E}\left[\boldsymbol{g}_{k}^{H} \boldsymbol{v}_{k}\right]$. In performance analysis,

\footnotetext{
${ }^{2}$ Throughout this paper, all powers are defined as averages over all sources of randomness ( $\hat{\boldsymbol{G}}$ in this particular equation, since $\left\{\boldsymbol{v}_{k}\right\}$ depend on $\hat{\boldsymbol{G}}$ ). This convention is common in the massive MIMO literature. The reason is mostly mathematical convenience. In principle, somewhat increased performance could be obtained by defining a short-term measure of power and allocating powers between the coherence intervals. However, in massive MIMO, the gain of doing so is not appreciable in typical cases because by virtue of the channel hardening, $\|\hat{\boldsymbol{G}}\|^{2}$ fluctuates only slightly from one coherence interval to the next.
}

the effect of the gain error $\boldsymbol{g}_{k}^{H} \boldsymbol{v}_{k}-\mathrm{E}\left[\boldsymbol{g}_{k}^{H} \boldsymbol{v}_{k}\right]$ is then treated as additional effective noise. This is a common approach in the massive MIMO literature [17], [18], but it is not optimal. For example, in low-mobility scenarios where the resource cost of downlink pilots is negligible, it is known that the transmission of downlink pilots improves performance [19]. Also, practical systems may use downlink pilots for various other practical reasons; certain downlink reference signals are typically transmitted in all wireless systems to enable synchronization and acquisition. Finally, we note that it is possible for the terminal to obtain a better estimate of $\boldsymbol{g}_{k}^{H} \boldsymbol{v}_{k}$ than $\mathrm{E}\left[\boldsymbol{g}_{k}^{H} \boldsymbol{v}_{k}\right]$ by using blind gain estimation techniques [20].

By way of contrast, in case no CSI at the base station is available, then beamforming as in (1) is not meaningful. Instead, the transmitted vectors $\{\boldsymbol{x}(t)\}$ may be constructed using space-time coding.

\section{JOINT BEAMFORMING AND BROADCASTING}

With joint beamforming and broadcasting (JBB), the base station simultaneously beamforms to $K$ B-terminals for which it has CSI, and broadcasts information aimed at the Oterminals. The fundamental feature of massive MIMO that makes this possible is that with $M$ antennas and beamforming to $K$ terminals, there are $M-K$ unused degrees of freedom. With JBB, the $M-K$ excess degrees of freedom are exploited by transmitting the broadcast information in a subspace orthogonal to the channel collectively seen by the $K$ B-terminals.

In detail, consider the transmission of $\boldsymbol{x}(t)$ on the downlink. The $k$ th B-terminal receives the following at time $t$ :

$$
y_{k}(t)=\boldsymbol{g}_{k}^{H} \boldsymbol{x}(t)+w_{k}(t),
$$

where $w_{k}(t)$ is noise, assumed to be $C N(0,1)$ here. Clearly, any part of the transmitted vector $\boldsymbol{x}(t)$ which falls in the nullspace of the following matrix:

$$
\boldsymbol{G}^{H} \triangleq\left[\boldsymbol{g}_{1}, \ldots, \boldsymbol{g}_{K}\right]^{H}
$$

will be invisible to all B-terminals. Hence, to $\boldsymbol{x}(t)$ formed as in (1), the base station may add any vector that lies in the nullspace of $G^{H}$. In particular, the base station may add broadcasting information aimed at the O-terminals. Since the base station does not have CSI to these O-terminals, it cannot beamform to them. However, it can use space-time coding.

In general, $\boldsymbol{G}$ will not be perfectly known at the base station. We assume that the base station has an estimate $\hat{\boldsymbol{G}}$ of $\boldsymbol{G}$. Let $\{\boldsymbol{z}(t)\}$ be a sequence of $M$-vectors intended for the Oterminals. Instead of (1), the base station then transmits at time $t$ the sum of two terms

$$
\boldsymbol{x}(t)=\sqrt{\rho_{b}} \cdot\left(\sum_{k=1}^{K} \boldsymbol{v}_{k} s_{k}(t)\right)+\sqrt{\rho_{o}} \cdot \Pi_{\hat{\boldsymbol{G}}}^{\perp} \boldsymbol{z}(t),
$$

where $\boldsymbol{z}(t)$ is normalized such that

$$
\mathrm{E}\left[\left\|\Pi_{\hat{\mathbf{G}}}^{\perp} \boldsymbol{z}(t)\right\|^{2}\right]=1 \text {. }
$$

\footnotetext{
${ }^{3}$ Throughout, $\Pi_{\boldsymbol{X}}^{\perp} \triangleq \boldsymbol{I}-\Pi_{\boldsymbol{X}}$, where $\Pi_{\boldsymbol{X}} \triangleq \boldsymbol{X}\left(\boldsymbol{X}^{H} \boldsymbol{X}\right)^{-1} \boldsymbol{X}^{H}$ denotes the projection onto the column space of $\boldsymbol{X}$.
} 
The first term of (5) represents data beamformed to the Bterminals and the second term represents broadcasting information aimed at the O-terminals. These two terms are statistically uncorrelated. The constants $\rho_{b}$ and $\rho_{o}$ represent the powers spent on the B-terminals and the O-terminals, and

$$
\rho_{d} \triangleq \rho_{b}+\rho_{o}
$$

is the total downlink power.

If $\hat{\boldsymbol{G}}$ is an accurate estimate of $\boldsymbol{G}$, then

$$
\boldsymbol{g}_{k}^{H} \Pi_{\hat{\boldsymbol{G}}}^{\perp} \approx \mathbf{0}
$$

for all $k$, so the B-terminals will not see significant interference arising from signals aimed at the O-terminals. The O-terminals will, however, see interference from the beamformed transmission aimed at the B-terminals.

\section{COnstruction of $\boldsymbol{z}(t)$}

$\mathrm{OA}$ is a special case when some resources are set aside for only transmission to the O-terminals and on these resources, $\boldsymbol{x}(t)=\sqrt{\rho_{o}} \cdot \boldsymbol{z}(t)$. Let $\boldsymbol{h}$ represent the channel between the array and an O-terminal. Both with OA and JBB, the Oterminals will not know $\boldsymbol{h}$ and hence the transmission aimed at the O-terminals, encoded in $\{\boldsymbol{z}(t)\}$, must be noncoherent or include pilots. With JBB, an O-terminal will not see the effect of the projection $\Pi \frac{\perp}{\hat{G}}$ explicitly. Instead, the O-terminal effectively sees $\boldsymbol{z}(t)$ transmitted over a channel with response $\Pi \frac{\perp}{\hat{G}} \boldsymbol{h}$. The vector $\boldsymbol{h}$ will be unknown to the O-terminal anyway, and so will be $\Pi_{\hat{\boldsymbol{G}}}^{\perp} \boldsymbol{h}$.

Henceforth, we assume that $\boldsymbol{z}(t)$ is confined to a subspace of dimension $M^{\prime}$, where $M^{\prime} \leq M$. Then we can write

$$
\boldsymbol{z}(t)=\boldsymbol{U} \boldsymbol{q}(t)
$$

for some $M^{\prime}$-vector $\boldsymbol{q}(t)$ that consists of encoded information to the O-terminals, where $\boldsymbol{U}$ is a semi-unitary $M \times M^{\prime}$ matrix; $\boldsymbol{U}^{H} \boldsymbol{U}=\boldsymbol{I}$. As a possible special case, $M^{\prime}=M$ and then, we may take $\boldsymbol{U}=\boldsymbol{I}$ without loss of generality. As another (albeit uninteresting) special case, $M^{\prime}=1$, which corresponds to "beamforming" with a channel-independent beamforming vector given by the sole column of $\boldsymbol{U}$. The matrix $\boldsymbol{U}$ is unknown to the O-terminals. We discuss some specifics of the choice of $\boldsymbol{U}$ later in this section.

The idea of confining $\boldsymbol{z}(t)$ to lie in a low-dimensional subspace was independently proposed by several authors [5], [6]. The motivation is that without this structure $\{\boldsymbol{z}(t)\}$ would have to contain $M$ pilot vectors. If $M$ is comparable to $\tau_{c}$ then a very large fraction of the downlink resources would have to be spent on pilots. This situation may well arise in massive MIMO: Consider an $M=100$-antenna array serving a suburban environment using a $2 \mathrm{GHz}$ carrier with 1 ms coherence time and $200 \mathrm{kHz}$ coherence bandwidth; then $\tau_{c}=200$. If $M>\tau_{c}$, then downlink training would even be impossible. By confining $\boldsymbol{z}(t)$ to have the form in 9 , only $M^{\prime}$ downlink pilot vectors are needed. The constant $M^{\prime}$ can then be selected such that $M^{\prime} \ll \tau_{c}$.

Space-time coding in the $M^{\prime}$-dimensional subspace offers spatial diversity of order $M^{\prime}$. Therefore, in environments with no frequency or time diversity, $M^{\prime}$ should not be too small. Conversely, if there is sufficient time and frequency diversity (outer coding over many coherence intervals), not much performance is lost by confining $\boldsymbol{z}(t)$ to an $M^{\prime}$-dimensional subspace [5].

When $\boldsymbol{z}(t)$ is constructed according to 9 then $\boldsymbol{q}(t)$, rather than $\boldsymbol{z}(t)$, should be generated by space-time coding. Here we will assume that $\boldsymbol{q}(t)$ has independent $C N(0, \xi)$ elements, where $\xi$ is chosen such that (6) is satisfied. This is not necessarily optimal but serves as a sound starting point in order to analyze the potential of JBB. In practice, some variant of space-time block coding may be used, as suggested in [5].

In the case of JBB, we will assume that $\boldsymbol{U}$ depends on $\hat{\boldsymbol{G}}$ in such a way that $\Pi_{\hat{\boldsymbol{G}}} \underset{\boldsymbol{U}}{\boldsymbol{U}}=\boldsymbol{U}$. This assumption is made mainly for analytical convenience. In practice this requires $\boldsymbol{U}$ to be random and selected anew in each coherence interval, but this is no restriction as the effective channel seen by an O-terminal is unknown anyway. This assumption requires that $M^{\prime} \leq M-K$, otherwise $\boldsymbol{U}$ cannot fit into the nullspace of $\hat{\boldsymbol{G}}^{H}$.

In the case of OA, $\boldsymbol{U}$ may be either fixed or selected randomly in each coherence interval subject to the condition that $\boldsymbol{U}^{H} \boldsymbol{U}=\boldsymbol{I}$. There is no restriction on $M^{\prime}$; it may range from 1 to $M$. As far as the choice of $\boldsymbol{U}$ is concerned, OA can be handled as a special case by letting $K=0$ so that $\hat{\boldsymbol{G}}$ is empty and $\Pi \frac{\perp}{\hat{G}}=\boldsymbol{I}$.

Under the assumptions made,

$$
\begin{aligned}
\mathrm{E}\left[\left\|\Pi_{\hat{\boldsymbol{G}}}^{\perp} \boldsymbol{z}(t)\right\|^{2}\right] & =\mathrm{E}\left[\left\|\Pi_{\hat{\hat{\boldsymbol{G}}}}^{\perp} \boldsymbol{U} \boldsymbol{q}(t)\right\|^{2}\right] \\
& =\xi \cdot \mathrm{E}\left[\operatorname{Tr}\left[\boldsymbol{U}^{H} \Pi_{\hat{\boldsymbol{G}}}^{\perp} \boldsymbol{U}\right]\right] \\
& =\xi \cdot \mathrm{E}\left[\operatorname{Tr}\left[\boldsymbol{U}^{H} \boldsymbol{U}\right]\right]=\xi M^{\prime} .
\end{aligned}
$$

Hence, in order for (6) to be satisfied, we must have

$$
\xi=\frac{1}{M^{\prime}} \text {. }
$$

In independent Rayleigh fading, as we will see in the analysis in Sections $\mathrm{V}$ and $\mathrm{VI}$, the only assumptions needed on $\boldsymbol{U}$ are that $\boldsymbol{U}^{H} \boldsymbol{U}=\boldsymbol{I}$ and $\Pi_{\hat{\boldsymbol{G}}}^{+} \boldsymbol{U}=\boldsymbol{U}$. In practice, however, in case some terminals do not experience independent Rayleigh fading, it may be wise to randomize $\boldsymbol{U}$ as much as possible under these given constraints. To generate such a "maximally random" $\boldsymbol{U}$, one may first compute an arbitrary semi-unitary $M \times(M-K)$ matrix $\boldsymbol{Q}$ whose columns span the orthogonal complement of the column space of $\hat{\boldsymbol{G}}$. This matrix $\boldsymbol{Q}$ then satisfies $\boldsymbol{Q}^{H} \boldsymbol{Q}=\boldsymbol{I}$ and $\boldsymbol{Q} \boldsymbol{Q}^{H}=\Pi_{\hat{\hat{\boldsymbol{G}}}}$. Then, generate an isotropically distributed [21] $(M-K)^{\mathbf{G}} \times(M-K)$ random matrix $\Psi$. Finally, let $\boldsymbol{U}$ be the $M^{\prime}$ first columns of $\boldsymbol{Q} \Psi$.

One could also in principle, in case the fading is known to deviate from independent Rayleigh and the correlation structure is known, optimize $\boldsymbol{U}$ based on the available side information on the covariance of the O-terminal channels'. More sophisticated schemes that perform stochastic beamforming and space-time coding [22] could also be used. We do not pursue that possibility in this paper however, as it is 
unclear to what extent the correlation structure of the fading can be known. In particular, some O-terminals may be silent for a long time so that the base station has no correlation information to them; also, if there are many O-terminals with different channel correlation then there is no single one-fits-all correlation that would be representative for every O-terminal. In addition, it appears that no clean closed-form performance results emerge under such assumptions.

\section{Performance of JoInt BeAmForming And BROADCASTING}

In this section, we derive lower bounds on the capacity for the B-terminals and O-terminals when JBB is used. Modified versions of these formulas apply when $\mathrm{OA}$ is used; see Section VI. Throughout, we assume that the terminals are subject to independent Rayleigh fading. That is, $\left\{\boldsymbol{g}_{k}\right\}$ are independent, and each $\boldsymbol{g}_{k}$ has independent elements with distribution $C N\left(0, \beta_{k}\right)$ where $\beta_{k}$ represents the path loss of the $k$ th terminal.

\section{A. Performance for the B-Terminals}

1) Channel Estimates: We assume that estimates of the channels $\left\{\boldsymbol{g}_{k}\right\}$ have been obtained by the base station based on measurements on mutually orthogonal uplink pilot sequences transmitted by the terminals, as in [17] and [18]. These pilot sequences are $\tau_{p}^{u}$ symbols long, where $\tau_{c} \geq \tau_{p}^{u} \geq K$. The estimate of $\boldsymbol{g}_{k}$, for $k=1, \ldots, K$, can be written as

$$
\hat{\boldsymbol{g}}_{k}=\boldsymbol{g}_{k}+\tilde{\boldsymbol{g}}_{k},
$$

where $\tilde{\boldsymbol{g}}_{k}$ is the estimation error. If MMSE estimation is used, a straightforward calculation shows that $\hat{\boldsymbol{g}}_{k}$ and $\tilde{\boldsymbol{g}}_{k}$ are mutually uncorrelated, zero-mean Gaussian with covariances

$$
\begin{aligned}
& \mathrm{E}\left[\hat{\boldsymbol{g}}_{k} \hat{\boldsymbol{g}}_{k}^{H}\right]=\gamma_{k} \boldsymbol{I} \\
& \mathrm{E}\left[\tilde{\boldsymbol{g}}_{k} \tilde{\boldsymbol{g}}_{k}^{H}\right]=\left(\beta_{k}-\gamma_{k}\right) \boldsymbol{I},
\end{aligned}
$$

where we defined

$$
\gamma_{k} \triangleq \frac{\tau_{p}^{u} \rho_{u} \beta_{k}^{2}}{1+\tau_{p}^{u} \rho_{u} \beta_{k}},
$$

and where $\rho_{u}$ is the uplink SNR, defined as the SNR measured at any of the base station antennas if a terminal with $\beta_{k}=1$ transmits with unit power.

2) Beamforming: The $k$ th B-terminal receives the following at time $t$ :

$$
\begin{aligned}
y_{k}(t) & =\sqrt{\rho_{b}} \cdot \boldsymbol{g}_{k}^{H}\left(\sum_{k^{\prime}=1}^{K} \boldsymbol{v}_{k^{\prime}} s_{k^{\prime}}(t)\right) \\
& +\sqrt{\rho_{o}} \cdot \boldsymbol{g}_{k}^{H} \Pi_{\hat{\boldsymbol{G}}}^{\perp} \boldsymbol{U} \boldsymbol{q}(t)+w_{k}(t)
\end{aligned}
$$

where $w_{k}(t)$ is $C N(0,1)$ noise. The beamforming vectors $\left\{\boldsymbol{v}_{k}\right\}$ are computed based on estimates of $\left\{\boldsymbol{g}_{k}\right\}$ obtained in the uplink. Henceforth, we consider maximum-ratio (MR) and zero-forcing (ZF) processing. For MR,

$$
\boldsymbol{v}_{k}=\boldsymbol{v}_{k}^{\mathrm{MR}} \triangleq \sqrt{\frac{\eta_{k}}{M \gamma_{k}}} \hat{\boldsymbol{g}}_{k},
$$

and for ZF,

$$
\boldsymbol{v}_{k}=\boldsymbol{v}_{k}^{Z \mathrm{~F}} \triangleq\left[\sqrt{\eta_{k} \gamma_{k}(M-K)} \hat{\boldsymbol{G}}\left(\hat{\boldsymbol{G}}^{H} \hat{\boldsymbol{G}}\right)^{-1}\right]_{:, k}
$$

where $[\cdot]_{:, k}$ denotes the $k$ th column of a matrix. In (17) and (18), $\left\{\eta_{k}\right\}$ are power control parameters that satisfy

$$
\sum_{k=1}^{K} \eta_{k}=1 .
$$

(We assume that the base station always expends full power.) With $\left\{\eta_{k}\right\}$ chosen as in (19, $\left\{\boldsymbol{v}_{k}^{\mathrm{MR}}\right\}$ and $\left\{\boldsymbol{v}_{k}^{\mathrm{zF}}\right\}$ satisfy (2). In massive MIMO, only slow power control is used so $\left\{\eta_{k}\right\}$ depend only on the path losses $\left\{\beta_{k}\right\}$.

3) Achievable Rate: No downlink pilots are used, and instead, the B-terminals rely on channel hardening. Using (17) and $(18)$ we can rewrite 160 in terms of a "useful signal term" plus a sequence of mutually uncorrelated noise and interference terms, as follows.

- For MR beamforming:

$$
\begin{aligned}
y_{k}(t)= & \sqrt{\frac{\rho_{b} \eta_{k}}{M \gamma_{k}}} \cdot \mathrm{E}\left[\left\|\hat{\boldsymbol{g}}_{k}\right\|^{2}\right] s_{k}(t) \\
& +\sqrt{\frac{\rho_{b} \eta_{k}}{M \gamma_{k}}} \cdot\left(\left\|\hat{\boldsymbol{g}}_{k}\right\|^{2}-\mathrm{E}\left[\left\|\hat{\boldsymbol{g}}_{k}\right\|^{2}\right]\right) s_{k}(t) \\
& -\sqrt{\rho_{b}} \cdot \tilde{\boldsymbol{g}}_{k}^{H}\left(\sum_{k^{\prime}=1}^{K} \boldsymbol{v}_{k^{\prime}}^{\mathrm{MR}} s_{k^{\prime}}(t)\right) \\
& +\sqrt{\rho_{b}} \cdot \hat{\boldsymbol{g}}_{k}^{H}\left(\sum_{k^{\prime}=1, k^{\prime} \neq k}^{K} \sqrt{\eta_{k^{\prime}}} \boldsymbol{v}_{k^{\prime}}^{\mathrm{MR}} s_{k^{\prime}}(t)\right) \\
& +\sqrt{\rho_{o}} \cdot \boldsymbol{g}_{k}^{H} \Pi_{\hat{\boldsymbol{G}}}^{\perp} \boldsymbol{U} \boldsymbol{q}(t)+w_{k}(t) .
\end{aligned}
$$

The first term in 20 represents the useful signal and is equal to $s_{k}(t)$ weighted by a deterministic constant. The second term represents the channel gain uncertainty at the terminal. The third term stems from channel estimation errors. The fourth term (summation of $K-1$ terms) stems from intracell interference. The fifth term stems from transmissions aimed at the O-terminals, but which are partly seen by the $k$ th B-terminal since $\Pi_{\hat{G}}^{\perp} \neq \Pi_{G}^{\perp}$. The sixth term is the thermal noise. The variances of the first four terms are known from [17] and [18]. Details are omitted here. The variance of the fifth term, which is specific to JBB, is shown in Appendix A to be equal to

$$
\rho_{o} \cdot \mathrm{E}\left[\left|\boldsymbol{g}_{k}^{H} \Pi \frac{\perp}{\hat{\boldsymbol{G}}} \boldsymbol{U} \boldsymbol{q}(t)\right|^{2}\right]=\rho_{o}\left(\beta_{k}-\gamma_{k}\right) \text {. }
$$

(The expectation here is with respect to all sources of randomness; hence the result is a deterministic constant.) Hence, using arguments in [17], [18], [23] we have the following achievable rate for the $k$ th terminal:

$$
R_{k}^{\mathrm{MR}}=\log _{2}\left(1+\frac{M \rho_{b} \gamma_{k} \eta_{k}}{\rho_{b} \beta_{k}+\rho_{o}\left(\beta_{k}-\gamma_{k}\right)+1}\right) .
$$



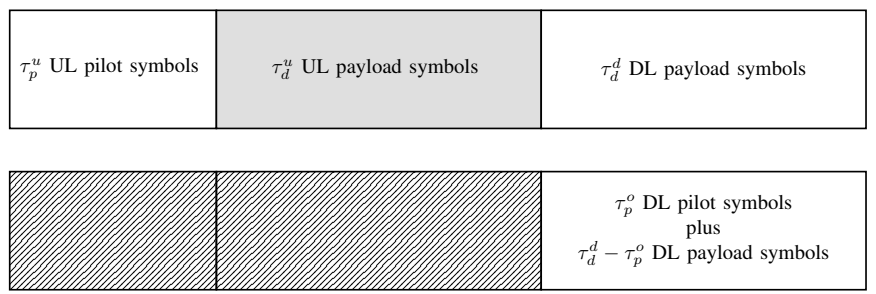

Fig. 1. Split of the $\tau_{c}$ symbols in a coherence interval with JBB, from the B-terminal perspective (upper) and the O-terminal perspective (lower).

- For ZF beamforming:

$$
\begin{aligned}
y_{k}(t)= & \sqrt{(M-K) \rho_{b} \gamma_{k} \eta_{k}} s_{k}(t) \\
& -\sqrt{\rho_{b}} \cdot \tilde{\boldsymbol{g}}_{k}^{H}\left(\sum_{k^{\prime}=1}^{K} \boldsymbol{v}_{k^{\prime}}^{\mathrm{ZF}} s_{k^{\prime}}(t)\right) \\
& +\sqrt{\rho_{o}} \cdot \boldsymbol{g}_{k}^{H} \Pi_{\hat{\boldsymbol{G}}}^{\perp} \boldsymbol{U} \boldsymbol{q}(t)+w_{k}(t) .
\end{aligned}
$$

Here, the first term represents the desired signal scaled by a deterministic constant. The second term stems from effects of channel estimation errors, the third term is leakage from the transmission aimed at the O-terminals and the fourth term is noise. The variances of the first two terms are known [17], [18] and the variance of the third term is the same as in the case of MR beamforming. The achievable rate is thus

$$
R_{k}^{\mathrm{zF}}=\log _{2}\left(1+\frac{(M-K) \rho_{b} \gamma_{k} \eta_{k}}{\left(\rho_{b}+\rho_{o}\right)\left(\beta_{k}-\gamma_{k}\right)+1}\right) .
$$

To compute a downlink net sum-spectral efficiency we assume that out of $\tau_{c}$ symbols in each coherence interval, $\tau_{p}^{u}$ symbols are used for uplink pilots (as above), $\tau_{d}^{u}$ symbols are used for uplink data and $\tau_{d}^{d}$ symbols are used for downlink data, where the uplink/downlink split is symmetric so that $\tau_{d}^{u}=\tau_{d}^{d}$; see Figure 11. In Figure 11, $\tau_{p}^{o}$ is the number of symbols out of the $\tau_{d}^{d}$ long downlink part of the coherence interval that are set aside for pilots to the O-terminals; to be explained in Section V-C2 The net downlink sum-spectral efficiency in the cell is then

$$
\begin{aligned}
R_{\mathrm{b}, \text { sum-net }} & \triangleq \frac{\tau_{d}^{d}}{\tau_{c}} \sum_{k=1}^{K} R_{k} \\
& =\frac{1}{2}\left(1-\frac{\tau_{p}^{u}}{\tau_{c}}\right) \sum_{k=1}^{K} R_{k} \quad \mathrm{~b} / \mathrm{s} / \mathrm{Hz} / \mathrm{cell},
\end{aligned}
$$

where $R_{k}$ is taken from (22) for MR and (24) for ZF. Note that we consider TDD operation and hence, to obtain rates all spectral efficiencies should be multiplied with the full system bandwidth used for both uplink and downlink.

\section{B. Power Control for the B-Terminals}

We adopt a max-min fairness power control policy that ensures that all B-terminals in the cell obtain the same rate. Such power control is useful to ensure a uniform quality-ofservice in the cell [24]. The resulting max-min optimal rate also is a neat proxy of the performance for the whole cell, expressed in terms only of the path loss profile $\left\{\beta_{k}\right\}$. To find the max-min operating point, $\left\{\eta_{k}\right\}$ should be selected such that 19 holds and such that $R_{k}^{\mathrm{MR}}=\bar{R}^{\mathrm{MR}, \mathrm{mm}}$ (for MR) respectively $\bar{R}_{k}^{\mathrm{ZF}}=\bar{R}^{\mathrm{zF}, \mathrm{mm}}$ (for $\mathrm{ZF}$ ) for some maximally large max-min optimal rates $\bar{R}^{\mathrm{Mr}, \mathrm{mm}}$ and $\bar{R}^{\mathrm{zF}, \mathrm{mm}}$ and for all $k$.

For MR, equating 22 to $\bar{R}^{\mathrm{MR}, \mathrm{mm}}$ and solving for $\eta_{k}$ yields

$$
\eta_{k}=\frac{\left(2^{\bar{R}^{\mathrm{MR}, \mathrm{mm}}}-1\right)\left(\rho_{b} \beta_{k}+\rho_{o}\left(\beta_{k}-\gamma_{k}\right)+1\right)}{M \rho_{b} \gamma_{k}} .
$$

Using the constraint 19$]$ we then conclude that

$$
\eta_{k}=\eta_{k}^{\mathrm{MR}} \triangleq \frac{\rho_{b} \beta_{k}+\rho_{o}\left(\beta_{k}-\gamma_{k}\right)+1}{\gamma_{k} \cdot \sum_{k^{\prime}=1}^{K} \frac{\rho_{b} \beta_{k^{\prime}}+\rho_{o}\left(\beta_{k^{\prime}}-\gamma_{k^{\prime}}\right)+1}{\gamma_{k^{\prime}}}} .
$$

A similar calculation for ZF yields

$$
\eta_{k}=\eta_{k}^{\mathrm{ZF}} \triangleq \frac{\left(\rho_{b}+\rho_{o}\right)\left(\beta_{k}-\gamma_{k}\right)+1}{\gamma_{k} \cdot \sum_{k^{\prime}=1}^{K} \frac{\left(\rho_{b}+\rho_{o}\right)\left(\beta_{k^{\prime}}-\gamma_{k^{\prime}}\right)+1}{\gamma_{k^{\prime}}}} .
$$

Note that $\left\{\eta_{k}^{\mathrm{MR}}\right\}$ and $\left\{\eta_{k}^{\mathrm{ZF}}\right\}$ depend on both $\rho_{b}$ and $\rho_{o}$. The max-min optimal rates (equal for all terminals in the cell) are, for MR respectively $\mathrm{ZF}$ :

$$
\begin{aligned}
& \bar{R}^{\mathrm{MR}, \mathrm{mm}}=\log _{2}\left(1+\frac{M \rho_{b}}{\sum_{k=1}^{K} \frac{\rho_{b} \beta_{k}+\rho_{o}\left(\beta_{k}-\gamma_{k}\right)+1}{\gamma_{k}}}\right), \\
& \bar{R}^{\mathrm{z}, \mathrm{mm}}=\log _{2}\left(1+\frac{(M-K) \rho_{b}}{\sum_{k=1}^{K} \frac{\left(\rho_{b}+\rho_{o}\right)\left(\beta_{k}-\gamma_{k}\right)+1}{\gamma_{k}}}\right) .
\end{aligned}
$$

\section{Performance for the O-Terminals}

An O-terminal with channel response $h$ will receive the following at time $t$ :

$$
y_{o}(t)=\sqrt{\rho_{o}} \cdot \boldsymbol{h}_{e}^{H} \boldsymbol{q}(t)+\sqrt{\rho_{b}} \cdot \boldsymbol{h}^{H}\left(\sum_{k=1}^{K} \boldsymbol{v}_{k} s_{k}(t)\right)+w_{o}(t),
$$

where

$$
\boldsymbol{h}_{e} \triangleq \boldsymbol{U}^{H} \Pi_{\hat{\mathbf{G}}}^{\perp} \boldsymbol{h}=\boldsymbol{U}^{H} \boldsymbol{h}
$$

represents the effective channel through which the O-terminal sees the $M^{\prime}$-dimensional signal $\boldsymbol{q}(t)$. In (31), the first term represents the signal of interest, the second term is interference that stems from the beamformed transmissions, and $w_{o}(t)$ is $C N(0,1)$ noise. We assume that the O-terminal sees independent Rayleigh fading. Then

$$
\boldsymbol{h} \sim C N\left(\mathbf{0}, \boldsymbol{C}_{\boldsymbol{h}}\right)
$$

where $\boldsymbol{C}_{\boldsymbol{h}}=\beta_{o} \cdot \boldsymbol{I}$ and where $\beta_{o}$ is the path loss of the O-terminal. Then, $\boldsymbol{h}_{e}$ is zero-mean with covariance matrix

$$
\begin{aligned}
\mathrm{E}\left[\boldsymbol{h}_{e} \boldsymbol{h}_{e}^{H} \mid \hat{\boldsymbol{G}}\right] & =\beta_{o} \cdot \boldsymbol{U}^{H} \boldsymbol{U}=\beta_{o} \cdot \boldsymbol{I} \\
& =\mathrm{E}\left[\boldsymbol{h}_{e} \boldsymbol{h}_{e}^{H}\right] \triangleq \boldsymbol{C}_{\boldsymbol{h}_{e}} .
\end{aligned}
$$



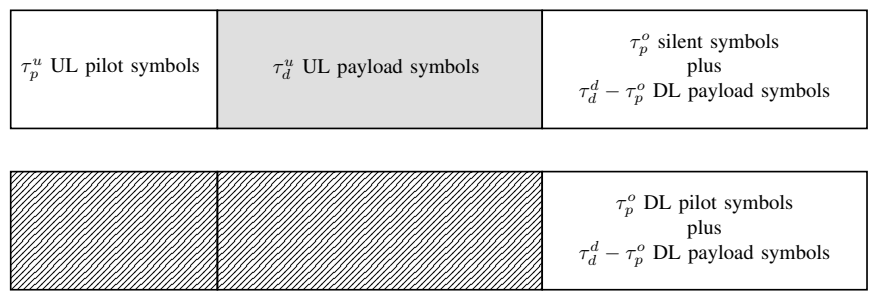

Fig. 2. Split of the $\tau_{c}$ symbols in a coherence interval with $\mathrm{JBB}^{\prime}$, from the B-terminal perspective (upper) and the O-terminal perspective (lower).

Recall, that $\boldsymbol{U}$ depends on $\hat{\boldsymbol{G}}$ as it is selected to lie in the nullspace of $\hat{\boldsymbol{G}}^{H}$. However, the covariance matrix $\boldsymbol{C}_{\boldsymbol{h}_{e}}$ is independent of $\hat{\boldsymbol{G}}$. Therefore, $\boldsymbol{h}_{e} \sim C N\left(\mathbf{0}, \boldsymbol{C}_{\boldsymbol{h}_{e}}\right)$.

1) Modified $J B B-J B B^{\prime}$ : When rigorously analyzing the capacity for the O-terminals, a technicality arises 4 We will consider a modified version of JBB where the B-terminals stay silent during the transmission of pilots to the O-terminals, see Figure 2. We give the name $\mathrm{JBB}^{\prime}$ to this modified version of $\mathrm{JBB}$, and denote all associated quantities with $(\cdot)^{\prime}$. In practice, the original JBB would likely be preferred over $\mathrm{JBB}^{\prime}$. The only motivation for introducing $\mathrm{JBB}^{\prime}$ is to facilitate the derivation of an achievable rate without approximations, as further discussed in Section VII

In order to spend the same amount of energy per coherence interval as with JBB in its original form as described in Section III for $\mathrm{JBB}^{\prime}, \rho_{b}$, must be replaced with

$$
\rho_{b}^{\prime} \triangleq \frac{\tau_{d}^{d}}{\tau_{d}^{d}-\tau_{p}^{o}} \cdot \rho_{b}=\frac{\frac{1}{2}\left(\tau_{c}-\tau_{p}^{u}\right)}{\frac{1}{2}\left(\tau_{c}-\tau_{p}^{u}\right)-\tau_{p}^{o}} \cdot \rho_{b} .
$$

With $\mathrm{JBB}^{\prime}$, the net downlink B-terminal sum-spectral efficiency is

$$
\begin{aligned}
R_{\mathrm{b}, \text { sum-net }}^{\prime} & \triangleq \frac{\tau_{d}^{d}-\tau_{p}^{o}}{\tau_{c}} \sum_{k=1}^{K} R_{k}^{\prime} \\
& =\frac{1}{2}\left(1-\frac{\tau_{p}^{u}+2 \tau_{p}^{o}}{\tau_{c}}\right) \sum_{k=1}^{K} R_{k}^{\prime} \quad \mathrm{b} / \mathrm{s} / \mathrm{Hz} / \text { cell. }
\end{aligned}
$$

While $\rho_{b}^{\prime}>\rho_{b}$, the extra loss in degrees of freedom in 35 renders $R_{\mathrm{b} \text {,sum-net }}^{\prime}<R_{\mathrm{b} \text {,sum-net }}$ in general. On the other hand, the O-terminal performance will be somewhat better when $\mathrm{JBB}^{\prime}$ is used instead of $\mathrm{JBB}$, since the O-terminals do not see interference on their pilots.

2) Pilot Phase: The transmission aimed at the O-terminals proceeds in two phases, first pilots and then payload.

The channel $\boldsymbol{h}_{e}$ is a priori unknown to the O-terminals, and must be estimated from pilots. Suppose that a string of $\tau_{p}^{o}$ downlink pilot vectors $\left\{\boldsymbol{q}_{p}(t)\right\}$ are transmitted to enable the O-terminals to learn $\boldsymbol{h}_{e}$. For good performance, these pilots should be orthonormal. If the energy spent per sample is the same during the pilot phase and the payload phase, $\left\{\boldsymbol{q}_{p}(t)\right\}$

\footnotetext{
${ }^{4}$ In preliminary work [4] we took a different approach that avoided this technicality. The resulting rate analysis for the O-terminals, however, was not entirely rigorous, although numerically it gave practically the same result as we derive here.
}

also should satisfy the power constraint (6). Hence, we assume that

$$
\sum_{t=1}^{\tau_{p}^{o}} \boldsymbol{q}_{p}(t) \boldsymbol{q}_{p}^{H}(t)=\frac{\tau_{p}^{o}}{M^{\prime}} \cdot \boldsymbol{I} .
$$

Equation 36 requires that $\tau_{c} \geq \tau_{p}^{o} \geq M^{\prime}$.

Note that in principle, the ratio between the energy per symbol during the pilot phase and the energy per symbol during the payload phase could be optimized, but we have not done that here. If $M^{\prime} \ll \tau_{c}$, the pre-log penalty of the pilot transmission is small and for performance analysis purposes the pilot power can be varied simply by tuning $\tau_{p}^{o}$, subject to $\tau_{c} \geq \tau_{p}^{o} \geq M^{\prime}$.

An O-terminal receives the $\tau_{p}^{o}$ noisy pilot symbols

$$
y_{o}(t)=\sqrt{\rho_{o}} \cdot \boldsymbol{h}_{e}^{H} \boldsymbol{q}_{p}(t)+w_{o}(t),
$$

where $w_{o}(t)$ is $C N(0,1)$ noise. (Due to the use of $\mathrm{JBB}^{\prime}$ instead of JBB, there is no interference from the transmission to the B-terminals here.) The O-terminal correlates $y_{o}(t)$ with the pilot sequence to obtain the following statistic:

$$
\boldsymbol{y}_{p} \triangleq \sum_{t=1}^{\tau_{p}^{o}} y_{o}^{*}(t) \boldsymbol{q}_{p}(t)=\frac{\tau_{p}^{o} \sqrt{\rho_{o}}}{M^{\prime}} \cdot \boldsymbol{h}_{e}+\boldsymbol{n}_{p}
$$

where

$$
\boldsymbol{n}_{p} \triangleq \sum_{t=1}^{\tau_{p}^{o}} w_{o}^{*}(t) \boldsymbol{q}_{p}(t)
$$

has zero mean and covariance

$$
\begin{aligned}
\boldsymbol{C}_{\boldsymbol{n}_{p}} & =\mathrm{E}\left[\boldsymbol{n}_{p} \boldsymbol{n}_{p}^{H}\right] \\
& =\mathrm{E}\left[\sum_{t=1}^{\tau_{p}^{o}} \sum_{t^{\prime}=1}^{\tau_{p}^{o}} w_{o}^{*}(t) w_{o}\left(t^{\prime}\right) \boldsymbol{q}_{p}(t) \boldsymbol{q}_{p}^{H}\left(t^{\prime}\right)\right] \\
& =\frac{\tau_{p}^{o}}{M^{\prime}} \cdot \boldsymbol{I} .
\end{aligned}
$$

From $\boldsymbol{y}_{p}$, the O-terminal can compute the MMSE estimate of $\boldsymbol{h}_{e}$ :

$$
\hat{\boldsymbol{h}}_{e}=\mathrm{E}\left[\boldsymbol{h}_{e} \mid \boldsymbol{y}_{p}\right]=\frac{M^{\prime} \sqrt{\rho_{o}} \beta_{o}}{M^{\prime}+\tau_{p}^{o} \rho_{o} \beta_{o}} \boldsymbol{y}_{p} .
$$

The estimation error $\tilde{\boldsymbol{h}}_{e} \triangleq \hat{\boldsymbol{h}}_{e}-\boldsymbol{h}_{e}$ and the estimate $\hat{\boldsymbol{h}}_{e}$ are uncorrelated and have covariances

$$
\begin{aligned}
& \boldsymbol{C}_{\tilde{\boldsymbol{h}}_{e}}=\mathrm{E}\left[\tilde{\boldsymbol{h}}_{e} \tilde{\boldsymbol{h}}_{e}^{H}\right]=\frac{M^{\prime} \beta_{o}}{M^{\prime}+\tau_{p}^{o} \rho_{o} \beta_{o}} \cdot \boldsymbol{I}, \\
& \boldsymbol{C}_{\hat{\boldsymbol{h}}_{e}}=\mathrm{E}\left[\hat{\boldsymbol{h}}_{e} \hat{\boldsymbol{h}}_{e}^{H}\right]=\frac{\tau_{p}^{o} \rho_{o} \beta_{o}^{2}}{M^{\prime}+\tau_{p}^{o} \rho_{o} \beta_{o}} \cdot \boldsymbol{I} .
\end{aligned}
$$

Since all quantities are jointly Gaussian, $\tilde{\boldsymbol{h}}_{e}$ and $\hat{\boldsymbol{h}}_{e}$ are independent. 
3) Payload Phase: Next, the O-terminal receives $\tau_{d}^{d}-\tau_{p}^{o}$ payload symbols. For these symbols, we have from (31) that

$$
\begin{aligned}
y_{o}(t) & =\sqrt{\rho_{o}} \cdot \hat{\boldsymbol{h}}_{e}^{H} \boldsymbol{q}(t)-\sqrt{\rho_{o}} \cdot \tilde{\boldsymbol{h}}_{e}^{H} \boldsymbol{q}(t) \\
& +\sqrt{\rho_{b}^{\prime}} \cdot \boldsymbol{h}^{H}\left(\sum_{k=1}^{K} \boldsymbol{v}_{k} s_{k}(t)\right)+w_{o}(t) .
\end{aligned}
$$

In (43), the first term represents the useful signal, the second term stems from channel estimation errors at the O-terminal, the third term comprises interference from transmissions aimed at the B-terminals, and $w_{o}(t)$ is $C N(0,1)$ noise.

All terms in (43) are mutually uncorrelated. Conditioned on $\hat{\boldsymbol{h}}_{e}$, the O-terminal sees the signal $\boldsymbol{q}(t)$ transmitted over a fixed, known channel $\hat{\boldsymbol{h}}_{e}$, embedded in additive uncorrelated (nonGaussian) noise. The distribution of the additive uncorrelated noise depends on $\hat{\boldsymbol{h}}_{e}$. However, $\hat{\boldsymbol{h}}_{e}$ is known to the O-terminal. Hence, we must compute the variances of all terms in (43) conditioned on $\hat{\boldsymbol{h}}_{e}$ :

- The conditional received power is

$$
\begin{aligned}
& \rho_{o} \cdot \mathrm{E}\left[\left|\hat{\boldsymbol{h}}_{e}^{H} \boldsymbol{q}(t)\right|^{2} \mid \hat{\boldsymbol{h}}_{e}\right] \\
& =\frac{\rho_{o}}{M^{\prime}} \cdot \mathrm{E}\left[\left\|\hat{\boldsymbol{h}}_{e}\right\|^{2} \mid \hat{\boldsymbol{h}}_{e}\right] \\
& =\frac{\rho_{o}}{M^{\prime}} \cdot\left\|\hat{\boldsymbol{h}}_{e}\right\|^{2} .
\end{aligned}
$$

- Since $\hat{\boldsymbol{h}}_{e}$ and $\tilde{\boldsymbol{h}}_{e}$ are independent, the second term of 43 has conditional variance

$$
\begin{aligned}
V_{1} & \triangleq \rho_{o} \cdot \mathrm{E}\left[\left|\tilde{\boldsymbol{h}}_{e}^{H} \boldsymbol{q}(t)\right|^{2} \mid \hat{\boldsymbol{h}}_{e}\right] \\
& =\frac{\rho_{o}}{M^{\prime}} \cdot \mathrm{E}\left[\left\|\tilde{\boldsymbol{h}}_{e}\right\|^{2} \mid \hat{\boldsymbol{h}}_{e}\right] \\
& =\frac{\rho_{o}}{M^{\prime}} \cdot \mathrm{E}\left[\left\|\tilde{\boldsymbol{h}}_{e}\right\|^{2}\right] \\
& =\frac{\rho_{o}}{M^{\prime}} \cdot \operatorname{Tr}\left[\boldsymbol{C}_{\tilde{\boldsymbol{h}}_{e}}\right] \\
& =\frac{M^{\prime} \rho_{o} \beta_{o}}{M^{\prime}+\tau_{p}^{o} \rho_{o} \beta_{o}},
\end{aligned}
$$

independently of $\hat{\boldsymbol{h}}_{e}$.

- The third term of 43 must be handled judiciously, due to the interdependence of $\boldsymbol{h}$ and $\hat{\boldsymbol{h}}_{e}$. First note that conditioned on $\hat{\boldsymbol{G}}, \boldsymbol{U}$ is fixed, so from 38 and $41,, \hat{\boldsymbol{h}}_{e}$ and $h$ are jointly Gaussian with zero means and crosscovariance

$$
\mathrm{E}\left[\boldsymbol{h} \hat{\boldsymbol{h}}_{e}^{H} \mid \hat{\boldsymbol{G}}\right]=\frac{\tau_{p}^{o} \rho_{o} \beta_{o}^{2}}{M^{\prime}+\tau_{p}^{o} \rho_{o} \beta_{o}} \cdot \boldsymbol{U}
$$

It follows that (see, e.g., [25, Lemma 2.4.1])

$$
\begin{aligned}
\mathrm{E}\left[\boldsymbol{h} \boldsymbol{h}^{H} \mid \hat{\boldsymbol{h}}_{e}, \hat{\boldsymbol{G}}\right] & =\boldsymbol{C}_{\boldsymbol{h}}-\mathrm{E}\left[\boldsymbol{h} \hat{\boldsymbol{h}}_{e}^{H} \mid \hat{\boldsymbol{G}}\right] \cdot \boldsymbol{C}_{\hat{\boldsymbol{h}}_{e}}^{-1} \cdot \mathrm{E}\left[\hat{\boldsymbol{h}}_{e} \boldsymbol{h}^{H} \mid \hat{\boldsymbol{G}}\right] \\
& =\beta_{o} \cdot \boldsymbol{I}-\frac{\tau_{p}^{o} \rho_{o} \beta_{o}^{2}}{M^{\prime}+\tau_{p}^{o} \rho_{o} \beta_{o}} \cdot \boldsymbol{U} \boldsymbol{U}^{H}
\end{aligned}
$$

In 447 we used that $\mathrm{E}\left[\hat{\boldsymbol{h}}_{e} \hat{\boldsymbol{h}}_{e}^{H} \mid \hat{\boldsymbol{G}}\right]=\mathrm{E}\left[\hat{\boldsymbol{h}}_{e} \hat{\boldsymbol{h}}_{e}^{H}\right]=\boldsymbol{C}_{\hat{\boldsymbol{h}}_{e}}$, similarly to in (33). Hence, the third term of (43) has conditional variance

$$
\begin{aligned}
& V_{2} \triangleq \rho_{b}^{\prime} \cdot \mathrm{E}\left[\left|\sum_{k=1}^{K} \boldsymbol{h}^{H} \boldsymbol{v}_{k} s_{k}(t)\right|^{2} \mid \hat{\boldsymbol{h}}_{e}\right] \\
& =\rho_{b}^{\prime} \cdot \mathrm{E}\left[\sum_{k=1}^{K} \boldsymbol{v}_{k}^{H} \boldsymbol{h} \boldsymbol{h}^{H} \boldsymbol{v}_{k} \mid \hat{\boldsymbol{h}}_{e}\right] \\
& =\rho_{b}^{\prime} \cdot \mathrm{E}\left[\mathrm{E}\left[\sum_{k=1}^{K} \boldsymbol{v}_{k}^{H} \boldsymbol{h} \boldsymbol{h}^{H} \boldsymbol{v}_{k} \mid \hat{\boldsymbol{h}}_{e}, \hat{\boldsymbol{G}}\right] \mid \hat{\boldsymbol{h}}_{e}\right] \\
& =\rho_{b}^{\prime} \cdot \mathrm{E}\left[\sum_{k=1}^{K} \boldsymbol{v}_{k}^{H} \mathrm{E}\left[\boldsymbol{h} \boldsymbol{h}^{H} \mid \hat{\boldsymbol{h}}_{e}, \hat{\boldsymbol{G}}\right] \boldsymbol{v}_{k} \mid \hat{\boldsymbol{h}}_{e}\right] \\
& =\rho_{b}^{\prime} \cdot\left(\beta_{o} \cdot \mathrm{E}\left[\sum_{k=1}^{K}\left\|\boldsymbol{v}_{k}\right\|^{2}\right]\right. \\
& \left.-\frac{\tau_{p}^{o} \rho_{o} \beta_{o}^{2}}{M^{\prime}+\tau_{p}^{o} \rho_{o} \beta_{o}} \cdot \mathrm{E}\left[\sum_{k=1}^{K} \boldsymbol{v}_{k}^{H} \boldsymbol{U} \boldsymbol{U}^{H} \boldsymbol{v}_{k} \mid \hat{\boldsymbol{h}}_{e}\right]\right) \\
& =\rho_{b}^{\prime} \beta_{o},
\end{aligned}
$$

independently of $\hat{\boldsymbol{h}}_{e}$. In 48 we used 2 and the fact that $\boldsymbol{U}^{H} \boldsymbol{v}_{k}=\mathbf{0}$ for all $k$ since $\boldsymbol{U}^{H} \hat{\boldsymbol{G}}=\mathbf{0}$ by construction; see (17) and (18). We also used that $\mathrm{E}\left[\sum_{k=1}^{K}\left\|\boldsymbol{v}_{k}\right\|^{2} \mid \hat{\boldsymbol{h}}_{e}\right]=\mathrm{E}\left[\sum_{k=1}^{K}\left\|\boldsymbol{v}_{k}\right\|^{2}\right]$, as the distribution of $\boldsymbol{U}^{H} \boldsymbol{h}$ conditioned on $\boldsymbol{U}$ is the same for all $\boldsymbol{U}$. In (48), when double expectations appear, the inner expectation is conditioned on $\hat{\boldsymbol{G}}$ and $\hat{\boldsymbol{h}}_{e}$, and the outer expectation is with respect to $\hat{\boldsymbol{G}}$ conditioned on $\hat{\boldsymbol{h}}_{e}$.

A lower capacity bound is obtained by assuming that the uncorrelated effective noise in (43) is Gaussian. Averaging over $\hat{\boldsymbol{h}}_{e}$ gives the following achievable rate for the O-terminal:

$$
\begin{aligned}
R_{o} & \triangleq \mathrm{E}\left[\log _{2}\left(1+\frac{\frac{\rho_{o}}{M^{\prime}} \cdot\left\|\hat{\boldsymbol{h}}_{e}\right\|^{2}}{V_{1}+V_{2}+1}\right)\right] \\
& =\mathrm{E}\left[\log _{2}\left(1+\frac{\frac{\rho_{o}}{M^{\prime}} \cdot\left\|\hat{\boldsymbol{h}}_{e}\right\|^{2}}{\frac{M^{\prime} \rho_{o} \beta_{o}}{M^{\prime}+\tau_{p}^{o} \rho_{o} \beta_{o}}+\rho_{b}^{\prime} \beta_{o}+1}\right)\right]
\end{aligned}
$$

In 49, the expectation is with respect to $\hat{\boldsymbol{h}}_{e}$. Since the Oterminal knows $\hat{\boldsymbol{h}}_{e}$, this average can be interpreted as an ergodic achievable rate. This rate only has a meaning if there is coding across multiple coherence intervals that see independent fading.

The expectation in (49) can be calculated in closed form [26. Theorem II.1], however, the result contains exponential integral functions of higher order and is difficult to interpret intuitively. To obtain a simple closed-form bound, we use the fact that if $\boldsymbol{\psi}$ is an $M^{\prime}$-vector with independent $C N(0,1)$ elements, then for any $\alpha>0$,

$$
\begin{aligned}
& \mathrm{E}\left[\log _{2}\left(1+\alpha\|\boldsymbol{\psi}\|^{2}\right)\right] \\
& \geq \log _{2}\left(1+\frac{\alpha}{\mathrm{E}\left[\frac{1}{\|\boldsymbol{\psi}\|^{2}}\right]}\right) \\
& =\log _{2}\left(1+\left(M^{\prime}-1\right) \alpha\right) .
\end{aligned}
$$


The first step in (50) follows from Jensen's inequality and the second step from a random matrix theory result $[27$. Lemma 2.10]. Since $\hat{\boldsymbol{h}}_{e}$ has independent Gaussian elements with variance

$$
\frac{\tau_{p}^{o} \rho_{o} \beta_{o}^{2}}{M^{\prime}+\tau_{p}^{o} \rho_{o} \beta_{o}}
$$

using (50) on 49, yields

$$
R_{o} \geq \log _{2}\left(1+\frac{\frac{M^{\prime}-1}{M^{\prime}} \cdot \rho_{o} \beta_{o} \cdot \frac{\tau_{p}^{o} \rho_{o} \beta_{o}}{M^{\prime}+\tau_{p}^{o} \rho_{o} \beta_{o}}}{\rho_{o} \beta_{o} \cdot \frac{M^{\prime}}{M^{\prime}+\tau_{p}^{o} \rho_{o} \beta_{o}}+\rho_{b}^{\prime} \beta_{o}+1}\right) .
$$

The inequality may not be tight if $M^{\prime}$ is small, but if $M^{\prime}$ is on the order of ten, or so, (52) should be not only a bound but also a reasonable approximation.

Taking into account the bandwidth cost of channel training, the net rate for an O-terminal is

$$
R_{o, \text { net }} \triangleq \frac{\tau_{d}^{d}-\tau_{p}^{o}}{\tau_{c}} \cdot R_{o}=\frac{1}{2}\left(1-\frac{\tau_{p}^{u}+2 \tau_{p}^{o}}{\tau_{c}}\right) \cdot R_{o}
$$

$\mathrm{b} / \mathrm{s} / \mathrm{Hz}$.

\section{Performance of Orthogonal Access}

Next we consider the option of orthogonal access (OA), where transmissions to the B-terminals and the O-terminals take place on orthogonal resources. Let $\epsilon$ be the fraction of the available coherence intervals that are used for transmission to the O-terminals so that $1-\epsilon$ is the fraction that remains for transmission to the B-terminals. Also, let $\rho_{b}^{\mathrm{OA}}$ and $\rho_{o}^{\mathrm{OA}}$ be the powers spent on the B- respectively O-terminals with OA. Generally, in what follows, the superscript $(\cdot)^{\mathrm{JBB}}$ will be used to denote quantities pertinent to $\mathrm{JBB}$, as derived in previous sections, and the superscript $(\cdot)^{\mathrm{OA}}$ will be used for OA.

\section{A. Performance for the B-Terminals}

The B-terminal rates with max-min fairness power control are obtained by setting $\rho_{o}=0$ and $\rho_{b}=\rho_{b}^{\mathrm{OA}}$ in 29 and 30 . and weighting the throughput by $1-\epsilon$ :

$$
\begin{aligned}
\bar{R}^{\mathrm{MR}, \mathrm{mm}, \mathrm{OA}} & =(1-\epsilon) \log _{2}\left(1+\frac{M \rho_{b}^{\mathrm{OA}}}{\sum_{k=1}^{K} \frac{\rho_{b}^{\mathrm{OA}} \beta_{k}+1}{\gamma_{k}}}\right) \\
\bar{R}^{\mathrm{ZF}, \mathrm{mm}, \mathrm{OA}} & =(1-\epsilon) \log _{2}\left(1+\frac{(M-K) \rho_{b}^{\mathrm{OA}}}{\sum_{k=1}^{K} \frac{\rho_{b}^{\mathrm{OA}}\left(\beta_{k}-\gamma_{k}\right)+1}{\gamma_{k}}}\right) .
\end{aligned}
$$

With OA there is no need for the B-terminals to be silent during the transmission of pilots to the O-terminals. Hence, the net sum-rates are obtained by multiplying $\bar{R}^{\mathrm{Mr}, \mathrm{mm}, \mathrm{OA}}$ and $\bar{R}^{\mathrm{ZF}, \mathrm{mm}, \mathrm{OA}}$ with

$$
\frac{1}{2}\left(1-\frac{\tau_{p}^{u}}{\tau_{c}}\right) \cdot K
$$

similarly to in 25). Also note that consequently, 54 and 55 contain $\rho_{b}$, not $\rho_{b}^{\prime}$.

\section{B. Performance for the O-Terminals}

The O-terminal rate is obtained by setting $\rho_{b}^{\prime}=0$ in 49 and weighting by $\epsilon$ :

$$
R_{o}^{\mathrm{OA}}=\epsilon \cdot \mathrm{E}\left[\log _{2}\left(1+\frac{\frac{\rho_{o}^{\mathrm{OA}}}{M^{\prime}} \cdot\left\|\hat{\boldsymbol{h}}_{e}\right\|^{2}}{\frac{M^{\prime} \rho_{o}^{\mathrm{OA}} \beta_{o}}{M^{\prime}+\tau_{p}^{o} \rho_{o}^{\mathrm{OA}} \beta_{o}}+1}\right)\right] .
$$

The corresponding bound is, from (52):

$$
R_{o}^{\mathrm{OA}} \geq \epsilon \cdot \log _{2}\left(1+\frac{\frac{M^{\prime}-1}{M^{\prime}} \cdot \rho_{o}^{\mathrm{OA}} \beta_{o} \cdot \frac{\tau_{p}^{o} \rho_{o}^{\mathrm{OA}} \beta_{o}}{M^{\prime}+\tau_{p}^{o} \rho_{o}^{\mathrm{OA}} \beta_{o}}}{\rho_{o}^{\mathrm{OA}} \beta_{o} \cdot \frac{M^{\prime}}{M^{\prime}+\tau_{p}^{o} \rho_{o}^{\mathrm{OA}} \beta_{o}}+1}\right) .
$$

Net-rates are obtained by multiplying with

$$
\frac{1}{2}\left(1-\frac{\tau_{p}^{u}+2 \tau_{p}^{o}}{\tau_{c}}\right)
$$

as in (53).

In order to make a fair comparison between $\mathrm{JBB}^{\prime}$ and $\mathrm{OA}, \epsilon$ must be chosen such that OA perform at its best. The find the optimal $\epsilon$ in this respect, we require that for a given "operating point" in terms of $\rho_{b}^{\mathrm{JBB}}$ and $\rho_{o}^{\mathrm{JBB}}$, the corresponding values of $\rho_{b}^{\mathrm{OA}}$ and $\rho_{o}^{\mathrm{OA}}$ must satisfy

$$
\rho_{b}^{\mathrm{JBB}}+\rho_{o}^{\mathrm{JBB}}=(1-\epsilon) \rho_{b}^{\mathrm{OA}}+\epsilon \rho_{o}^{\mathrm{OA}} .
$$

Equation 60 guarantees that the total energy spent in a coherence interval is the same in both cases. In order for OA to yield the same B-terminal performance as $\mathrm{JBB}^{\prime}$ does at this operating point, we require that

$$
\begin{aligned}
\bar{R}^{\mathrm{MR}, \mathrm{mm}, \mathrm{OA}} & =\bar{R}^{\mathrm{MR}, \mathrm{mm}, \mathrm{JBB}{ }^{\prime}}, \\
\text { respectively } \quad \bar{R}^{\mathrm{ZF}, \mathrm{mm}, \mathrm{OA}} & =\bar{R}^{\mathrm{ZF}, \mathrm{mm}, \mathrm{JBB}},
\end{aligned}
$$

for some $\epsilon, 0<\epsilon<1$. Given $\rho_{b}^{\mathrm{JBB}}, \rho_{o}^{\mathrm{JBB}}$ and $\epsilon$, solving (61) and 62 for $\rho_{b}^{\mathrm{OA}}$ we can determine how much is the B-terminal power needed with OA, as follows:

$$
\begin{aligned}
& \rho_{b}^{\mathrm{MR}, \mathrm{OA}}=\frac{\left(2^{\frac{\bar{R}^{\mathrm{MR}, \mathrm{mm}, \mathrm{JBB}{ }^{\prime}}}{1-\epsilon}}-1\right) \sum_{k=1}^{K} \frac{1}{\gamma_{k}}}{M-\left(2^{\frac{\bar{R}^{\mathrm{MR}, \mathrm{mm}, \mathrm{JBB} B^{\prime}}}{1-\epsilon}}-1\right) \sum_{k=1}^{K} \frac{\beta_{k}}{\gamma_{k}}}, \\
& \rho_{b}^{\mathrm{ZF}, \mathrm{OA}}=\frac{\left(2^{\frac{\bar{R}^{\mathrm{ZF}, \mathrm{mm}, \mathrm{JBB} B^{\prime}}}{1-\epsilon}}-1\right) \sum_{k=1}^{K} \frac{1}{\gamma_{k}}}{M-K-\left(2^{\frac{\bar{R}^{\mathrm{ZF}, \mathrm{mm}, \mathrm{JBB}}{ }^{\prime}}{1-\epsilon}}-1\right) \sum_{k=1}^{K} \frac{\beta_{k}-\gamma_{k}}{\gamma_{k}}} .
\end{aligned}
$$

Then, solving 60 with respect to $\rho_{o}^{\mathrm{OA}}$, subject to the constraint that $\rho_{o}^{\mathrm{OA}} \geq 0$, we can find how much power that remains to spend on the O-terminals. The solution to 60 may not exist, because of the requirement that $\rho_{o}^{\mathrm{OA}} \geq 0$. In case a solution exists, $R_{o}^{\mathrm{OA}}$ is given by (57), and in case no solution exists we set $R_{o}^{\mathrm{OA}}=0$. Next, for each operating point we find the value of $\epsilon, 0 \leq \epsilon \leq 1$, that maximizes $R_{o}^{\mathrm{OA}}$. We do not have a closed-form expression for this optimal $\epsilon$, and in the numerical examples it was chosen by a grid search from 0 to 1 . Typically, performance is not very sensitive to the choice of $\epsilon$. 
Taken together, the above-described procedure gives us, for any $\left(\rho_{b}^{\mathrm{JB}}, \rho_{o}^{\mathrm{JBB}}\right)$, the values of $\left(\rho_{b}^{\mathrm{OA}}, \rho_{o}^{\mathrm{OA}}\right)$ for which 60 and 61) respectively 62 hold, and for which $R_{o}^{\mathrm{OA}}$ is as large as possible.

\section{DISCUSSION}

The capacity bounds 29 and 30 for the B-terminal performance, along with the bound $(52)$ on the O-terminal performance, give insights into the impact of the various system parameters on performance:

- $M$ and $K$ substantially affect only the performance of the B-terminals, but not the performance of the O-terminals. JBB in principle works for any $M$ and $K(K<M)$. However, it underperforms OA unless $M$ is sufficiently large. This is the "massive MIMO" aspect of JBB.

- In terms of B-terminal performance, the leakage that occurs when projecting the O-terminal signals onto the nullspace of $\hat{\boldsymbol{G}}^{H}$, rather than that of $\boldsymbol{G}^{H}$, depends only on $\rho_{o}$ and on the quality of the channel state information (as characterized by $\gamma_{k}$ ). The better uplink SNR $\rho_{u}$, the closer is $\gamma_{k}$ to $\beta_{k}$ and the smaller is this leakage.

- In terms of O-terminal performance, unless the effects of channel estimation errors dominate, the performance is essentially determined by $\rho_{o}, \rho_{b}^{\prime}$ and $\beta_{o}$. Consider (52). For the effect of channel estimation errors to be negligible, we need

$$
\tau_{p}^{o} \gg \frac{M^{\prime}}{\rho_{o} \beta_{o}}
$$

so the number of downlink pilots must scale with $M^{\prime}-$ consistently with intuition.

A few other technical remarks are in order:

- For performance analysis, a modification (called $\mathrm{JBB}^{\prime}$ ) of JBB was considered, where the B-terminals are silent during the training phase of the O-terminals. We stress that this modification is not necessary, or even desired, if applying JBB in practice. It was only introduced in order to enable the calculation of a lower bound on ergodic capacity for the O-terminals.

The difficulty with a rigorous analysis of the original JBB scheme is, in more detail, the following. With the original JBB the received pilots in (37), will depend on $\hat{G}$ and on the (random) symbols transmitted to the Bterminals during the time when pilots are transmitted to the O-terminals. Hence the channel estimate $\hat{\boldsymbol{h}}_{e}$ will also depend on those quantities. This dependence must be taken into account when computing the conditional (on $\hat{\boldsymbol{h}}_{e}$ ) variances in 45 and $(48)$, which we were unable to obtain in closed form.

- Throughout, in order to understand and expose the tradeoffs associated with JBB at maximum possible depth, we have focused on a single-cell setup. In a multi-cell setup, additional interference will be present from other cells. This interference comprises among others so-called "pilot contamination" which is known to constitute an ultimate limitation in the sense that unlike all other interference, it does not go away even if $M \rightarrow \infty$ [1].

Using results known from, for example [28], one can show that the effects of these additional sources of interference, when deriving capacity lower bounds for the B-terminals, can be accounted for by scaling the numerator and augmenting the denominator inside the logarithm in 22) and 24) with additional deterministic terms. The rate expressions for the O-terminals could also be modified to take into account the effects of inter-cell interference. Hence, in principle, the analysis here could be extended to a multi-cell setup; however, a comprehensive performance evaluation would require serious system simulations which in turn requires judicious choices of power control policies, pilot reuse and allocation schemes, and terminal-base station association algorithms. We believe that such simulations could easily obscure the main points we wish to make in this paper. Hence, extensions of the performance evaluation to multicell setups have to be left for future work.

\section{NUMERICAL EXAMPLES}

JBB does not uniformly outperform OA, but there are many situations when it performs substantially better. Here, we provide some examples of such cases. With MR beamforming JBB almost always outperforms OA. Since JBB is as computationally demanding as ZF, we consider only ZF beamforming in the examples here. Due to the lack of availability of performance bounds for $\mathrm{JBB}$, in all comparisons we consider $\mathrm{JBB}^{\prime}$ instead of JBB, even though $\mathrm{JBB}$ is expected to perform somewhat better in practice. However, as in the derivations, we use $\left(\rho_{b}^{\mathrm{JBB}}, \rho_{o}^{\mathrm{JBB}}\right)$ to define the system operating point.

In the numerical examples, $K$ terminals were placed inside an annulus-shaped cell with outer radius 1 unit and inner radius 0.1 unit. A standard log-distance path loss model with exponent 4 was used. However, there was no shadow fading. Fast fading was modeled as Rayleigh and independent between the antennas. The length of the coherence interval was $\tau_{c}=500$ symbols, corresponding to mobile suburban radio access in the $2 \mathrm{GHz}$-band (2 $\mathrm{ms}$ coherence time; 250 $\mathrm{kHz}$ coherence bandwidth). The uplink cell-edge SNR was $\rho_{u}=-3 \mathrm{~dB}$. This SNR corresponds to a gross spectral efficiency of $\log _{2}\left(1+10^{-3 / 10}\right) \approx 0.6 \mathrm{~b} / \mathrm{s} / \mathrm{Hz}$ for a reference SISO AWGN link-however, owing to the large array gain, massive MIMO delivers good performance even at such low SNRs.

Performance for B-terminals was evaluated in terms of achievable net sum-rate with max-min power control. Performance for the O-terminals was evaluated in terms of net rate, assuming that the O-terminals are located at the cell border. Specifically, as functions of the total downlink power $\rho_{d}^{\mathrm{JBB}}=\rho_{b}^{\mathrm{JBB}}+\rho_{o}^{\mathrm{JBB}}$ and the power ratio $\rho_{o}^{\mathrm{JBB}} / \rho_{b}^{\mathrm{JBB}}$, we determine:

(i) The set of operating points for which $\mathrm{JBB}^{\prime}$ achieves a pre-determined net target sum-rate to the B-terminals of $R_{b, \text { sum-net }}^{*} \mathrm{~b} / \mathrm{s} / \mathrm{Hz}$ - that is, owing to the max-min power 
control, $R_{b, \text { sum-net }}^{*} / K \mathrm{~b} / \mathrm{s} / \mathrm{Hz}$ guaranteed to each one of the B-terminals. These are the black curves.

(ii) The set of operating points for which $\mathrm{JBB}^{\prime}$ delivers a predetermined net target rate of $R_{o, \text { net }}^{*} \mathrm{~b} / \mathrm{s} / \mathrm{Hz} /$ terminal to the O-terminals. These are the red curves.

(iii) The set of operating points for which there exist a resource split parameter $\epsilon$ and a feasible power allocation $\left(\rho_{b}^{\mathrm{OA}}, \rho_{o}^{\mathrm{OA}}\right)$ with which OA delivers the same Bterminal performance as does $\mathrm{JBB}^{\prime}$, and simultaneously a pre-determined O-terminal net target rate of $R_{o, \text { net }}^{*}$ $\mathrm{b} / \mathrm{s} / \mathrm{Hz} /$ terminal. These are the blue curves.

Figures 375 show concrete examples:

- Figure 3 . Here, $M=100$ antennas serve a single $(K=1)$ terminal. Both the B-terminal and the O-terminals are randomly located on the cell border. The target B-terminal rate is $2 \mathrm{~b} / \mathrm{s} / \mathrm{Hz}$ and the target O-terminal rate is 0.75 $\mathrm{b} / \mathrm{s} / \mathrm{Hz}^{5}$ A pilot sequence of length $\tau_{p}=10$ symbols was used in the uplink, which is easily afforded given the long channel coherence. In the downlink, somewhat arbitrarily, $M^{\prime}=7$ and $\tau_{p}^{o}=10$.

The selected operating point can be achieved in two ways: (i) using $\mathrm{JBB}^{\prime}$, and (ii) using OA. These two possibilities correspond to the following two intersection points between the curves in the figure: (i) when the curve for $2 \mathrm{~b} / \mathrm{s} / \mathrm{Hz}$ B-terminal performance intersects the curve for $0.75 \mathrm{~b} / \mathrm{s} / \mathrm{Hz}$ O-terminal performance with $\mathrm{JBB}^{\prime}$, and (ii) when the curve for $2 \mathrm{~b} / \mathrm{s} / \mathrm{Hz}$ B-terminal performance intersects the curve for $0.75 \mathrm{~b} / \mathrm{s} / \mathrm{Hz}$ O-terminal performance with OA. In terms of required total radiated power, $\mathrm{JBB}^{\prime}$ offers savings of about $3 \mathrm{~dB}$ compared to OA.

Note that at the operating point of interest, most of the radiated power is spent on the O-terminals: It is expensive to reach those terminals since no array gain is available.

- Figure 4. Here, $M=100$ antennas serve $K=10$ terminals. The B-terminals were dropped at random in the cell, yielding a path loss profile consisting of $K$ values $\left\{\beta_{k}\right\}$. The $\mathrm{O}$-terminals are at the cell border, with an additional fading margin of $10 \mathrm{~dB}$. This models a scenario in which the O-terminals are deeply shadowed and the base station has to expend significant resources in order to reach the O-terminals. The target B-terminal rate is $2 \mathrm{~b} / \mathrm{s} / \mathrm{Hz} /$ terminal $(20 \mathrm{~b} / \mathrm{s} / \mathrm{Hz}$ sum-rate) and the target O-terminal rate is $0.5 \mathrm{~b} / \mathrm{s} / \mathrm{Hz}$. A pilot sequence of length $\tau_{p}^{u}=30$ symbols is used in the uplink, that is, three symbols per terminal, which is afforded without problem given the long channel coherence. In the downlink, $M^{\prime}=7$ and $\tau_{p}^{o}=10$. The power saving of $\mathrm{JBB}^{\prime}$ compared to OA here is about $2.5 \mathrm{~dB}$.

- Figure 5. Here, $M=150$ antennas serve $K=30$ terminals randomly located in the cell. The O-terminals are at the cell border (without any extra fading margin). The

\footnotetext{
${ }^{5}$ Note that while these spectral efficiencies may seem low, they are twice as high during the time when transmission in the downlink actually takes place. For comparison with a frequency-division duplexing system, all numbers should be multiplied by the total bandwidth allocated for both uplink and downlink.
}

B-terminal target rate is $1.67 \mathrm{~b} / \mathrm{s} / \mathrm{Hz} /$ terminal $(50 \mathrm{~b} / \mathrm{s} / \mathrm{Hz}$ sum-rate) and the O-terminal target rate is $0.75 \mathrm{~b} / \mathrm{s} / \mathrm{Hz}$. In the uplink, $\tau_{p}^{u}=60$ pilot symbols are used and in the downlink, $M^{\prime}=7$ and $\tau_{p}^{o}=10$. The gain of $\mathrm{JBB}^{\prime}$ over $\mathrm{OA}$ is smaller here, but still tangible.

Note that the O-terminal rate $R_{o}$ is a monotonically decreasing function of the O-terminal path loss $\beta_{o}$. This can be seen from (52). Hence, the cell border is the worst possible location for an O-terminal so in that respect the examples in Figures 35 show worst-case performance. In practice, it could happen that the O-terminals are located closer to the base station. They could then be served with somewhat higher rate. However, the increase in rate is marginal in cases of interest. To exemplify, Figure 6 shows a variation of the result of Figure 3 , when the O-terminal is located halfway between the base station and the cell border. Qualitatively, Figure 6 is similar to Figure 3 . but a lower total power is required.

To provide additional insight, Table $\mathbb{I}$ shows for each of the examples in Figures 3 -5 and the two possible operating points, the following quantities:

- The optimal value of $\epsilon$ for OA, when applicable.

- The power of the received useful signal for the O-terminal relative to the thermal noise, that is, the numerator of (52).

- The strength of the effective noises that affect performance of the O-terminals relative to the thermal noise, that is, the first two terms in the denominator of (52).

From the table, we can infer that depending on the operating scenario, the main impairment is either thermal noise or interference from the B-terminal transmission; sufficient pilots are allocated on the downlink. Yet, the effects of channel estimation errors are not negligible.

As an additional illustration, Figure 7 shows the required B-terminal power $\rho_{b}$ for given O-terminal power $\rho_{o}$ in order to maintain a B-terminal sum-rate of $20 \mathrm{~b} / \mathrm{s} / \mathrm{Hz}$ with $M=100$ antennas and $K=10$ terminals (that is, $2 \mathrm{~b} / \mathrm{s} / \mathrm{Hz} /$ terminal). The channel coherence was $\tau_{c}=500$ symbols of which $\tau_{p}^{u}=$ 30 were spent on uplink pilots. Results are shown for different uplink pilot SNR $\rho_{u}$. It can be seen that the better uplink pilot quality, the more accurate channel state information is available to the B-terminals and the less B-terminal power is required to maintain the same rate. This is expected, because the larger $\rho_{u}$ is, the closer is $\gamma_{k}$ to $\beta_{k}$ and the less is the leakage power in 21).

\section{CONCLUSIONS}

The surplus of spatial degrees of freedom in massive MIMO makes it possible to "hide" signals in the channel nullspace, which terminals targeted by beamforming do not see. With joint beamforming and broadcasting (JBB), this opportunity is used to broadcast public information, aimed at terminals to which the base station does not have channel state information. Depending on the selected operating point, JBB can offer savings in radiated power in the order of $3 \mathrm{~dB}$ compared to orthogonal access. An additional, less obvious advantage of JBB is that the broadcast information is spread over all 


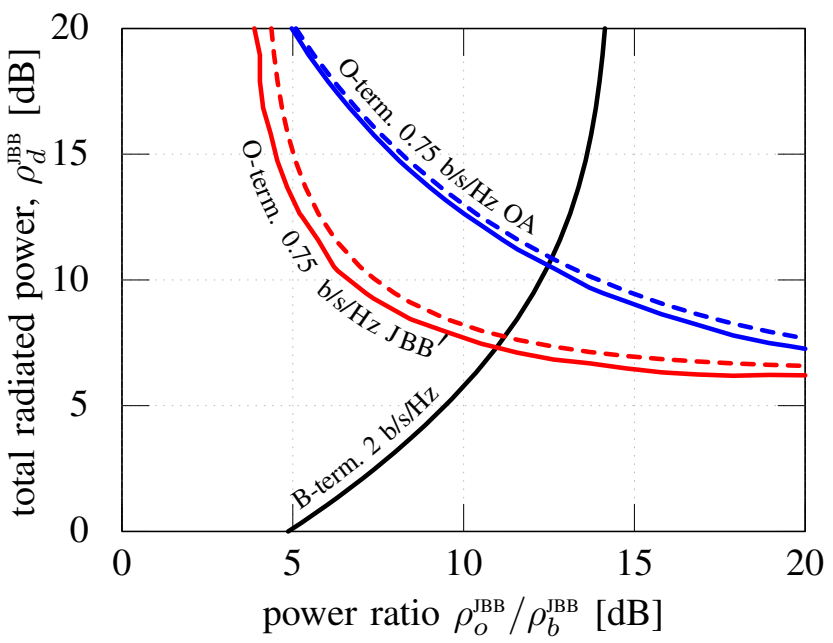

Fig. 3. Feasible operating points in terms of the total downlink power $\rho_{d}^{\mathrm{BB}}=\rho_{b}^{\mathrm{JBB}}+\rho_{o}^{\mathrm{JBB}}$ and the power ratio $\rho_{o}^{\mathrm{JBB}} / \rho_{b}^{\mathrm{JBB}}$, which yield a predetermined net rate for a B- and an O-terminal, both located at the cell border, for the case of $M=100$ antennas, $K=1 \mathrm{~B}$-terminal, $\tau_{c}=500$ symbols channel coherence, $\rho_{u}=-3 \mathrm{~dB}$ uplink SNR, $\tau_{p}^{u}=10$ uplink pilot symbols, dimensionality $M^{\prime}=7$ of the reduced channel, and $\tau_{p}^{o}=10$ downlink pilots. The black line represents the expression 35 for $\mathrm{B}$-terminal rate with $\mathrm{JBB}^{\prime}$ The solid red line represents the O-terminal rate with $\mathrm{JBB}^{\prime}, 49$ weighted to account for the pilot cost. The solid blue line represents the O-terminal rate with OA, 57) weighted to account for the pilot cost and optimized with respect to $\epsilon$. The red and blue dashed lines represent the corresponding closedform bounds 52) respectively (58) on the O-terminal rates.

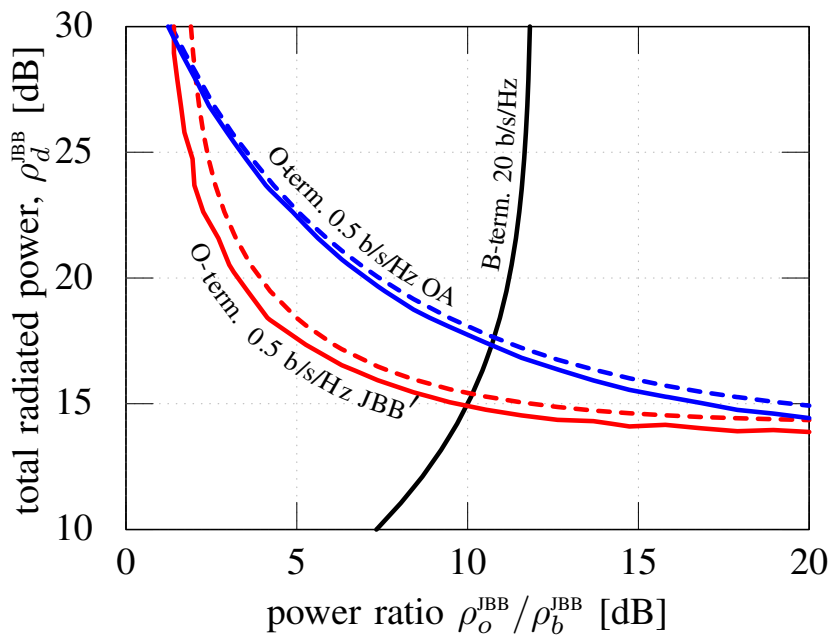

Fig. 4. Same as Figure 3 but for $M=100$ antennas serving $K=10$ B-terminals located at random in the cell. Here $\tau_{p}^{u}=30, \tau_{p}^{o}=10$ and $M^{\prime}=7$. The O-terminals were located on the cell border, with an additional shadow margin of $10 \mathrm{~dB}$

time-frequency resources, so the maximum possible time and frequency diversity is always exploited.

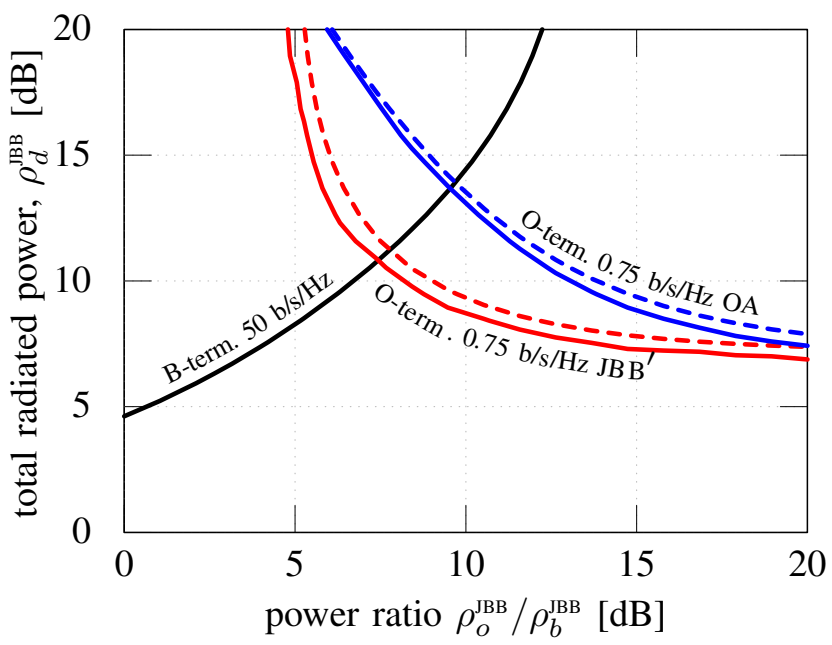

Fig. 5. Same as Figure 4 but for $M=150$ antennas serving $K=30$ terminals located at random in the cell. Here $\tau_{p}^{u}=60, \tau_{p}^{o}=10$ and $M^{\prime}=7$. The O-terminals were located on the cell border, with no additional shadow margin.

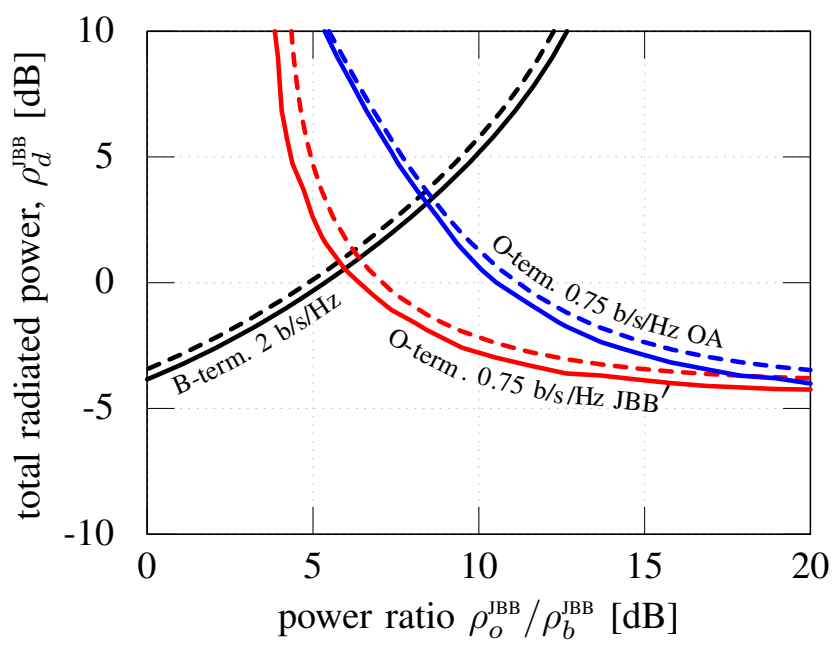

Fig. 6. Same as Figure 3 but here the O-terminals were located halfway between the base station and the cell border.

\section{APPENDIX A}

$$
\text { Calculation of E }\left[\left|\sqrt{\rho_{o}} \cdot \boldsymbol{g}_{k}^{H} \Pi_{\hat{\mathbf{G}}}^{\perp} \boldsymbol{U} \boldsymbol{q}(t)\right|^{2}\right]
$$

First note that

$$
\mathrm{E}\left[\boldsymbol{g}_{k} \boldsymbol{g}_{k}^{H} \mid \hat{\boldsymbol{G}}\right]=\hat{\boldsymbol{g}}_{k} \hat{\boldsymbol{g}}_{k}^{H}+\left(\beta_{k}-\gamma_{k}\right) \boldsymbol{I} .
$$




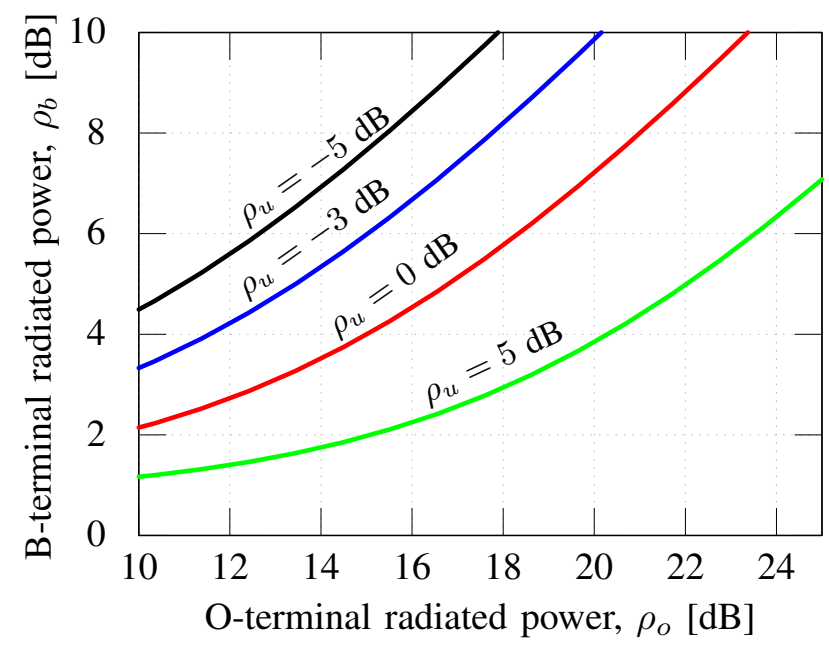

Fig. 7. Example of required B-terminal power $\rho_{b}$ for given O-terminal power $\rho_{o}$ in order to maintain a B-terminal sum-rate of $20 \mathrm{~b} / \mathrm{s} / \mathrm{Hz}$ with $M=100$ antennas and $K=10$ terminals, for different uplink pilot quality $\rho_{u}$. The channel coherence was $\tau_{c}=500$ symbols of which $\tau_{p}^{u}=30$ were spent on uplink pilots.

Therefore, since $\Pi_{\hat{\boldsymbol{G}}}^{\perp} \hat{\boldsymbol{g}}_{k}=\mathbf{0}$,

$$
\begin{aligned}
& \mathrm{E}\left[\left|\sqrt{\rho_{o}} \cdot \boldsymbol{g}_{k}^{H} \Pi_{\hat{\hat{G}}}^{\perp} \boldsymbol{U} \boldsymbol{q}(t)\right|^{2}\right] \\
= & \rho_{o} \cdot \mathrm{E}\left[\left|\boldsymbol{g}_{k}^{H} \Pi_{\hat{\boldsymbol{G}}}^{\perp} \boldsymbol{U} \boldsymbol{q}(t)\right|^{2}\right] \\
= & \frac{\rho_{o}}{M^{\prime}} \cdot \mathrm{E}\left[\operatorname{Tr}\left[\boldsymbol{U}^{H} \Pi_{\hat{\hat{G}}}^{\perp} \boldsymbol{g}_{k} \boldsymbol{g}_{k}^{H} \Pi_{\hat{\boldsymbol{G}}}^{\perp} \boldsymbol{U}\right]\right] \\
= & \frac{\rho_{o}}{M^{\prime}} \cdot \operatorname{Tr}\left[\mathrm{E}\left[\mathrm{E}\left[\boldsymbol{U}^{H} \Pi_{\hat{\hat{G}}}^{\perp} \boldsymbol{g}_{k} \boldsymbol{g}_{k}^{H} \Pi_{\hat{\hat{G}}}^{\perp} \boldsymbol{U} \mid \hat{\boldsymbol{G}}\right]\right]\right] \\
= & \frac{\rho_{o}}{M^{\prime}} \cdot \operatorname{Tr}\left[\mathrm{E}\left[\boldsymbol{U}^{H} \Pi_{\hat{\hat{G}}}^{\perp} \cdot \mathrm{E}\left[\boldsymbol{g}_{k} \boldsymbol{g}_{k}^{H} \mid \hat{\boldsymbol{G}}\right] \cdot \Pi_{\hat{\hat{G}}}^{\perp} \boldsymbol{U}\right]\right] \\
= & \frac{\rho_{o}}{M^{\prime}} \cdot \operatorname{Tr}\left[\mathrm{E}\left[\boldsymbol{U}^{H} \Pi_{\hat{\hat{G}}}^{\perp}\left(\hat{\boldsymbol{g}}_{k} \hat{\boldsymbol{g}}_{k}^{H}+\left(\beta_{k}-\gamma_{k}\right) \boldsymbol{I}\right) \Pi_{\hat{\hat{G}}}^{\perp} \boldsymbol{U}\right]\right] \\
= & \frac{\rho_{o}}{M^{\prime}}\left(\beta_{k}-\gamma_{k}\right) \cdot \operatorname{Tr}\left[\mathrm{E}\left[\boldsymbol{U}^{H} \Pi_{\hat{\hat{G}}}^{\perp} \boldsymbol{U}\right]\right] \\
= & \rho_{o}\left(\beta_{k}-\gamma_{k}\right) .
\end{aligned}
$$

After the third equality sign, double expectations appear. The inner expectation is with respect to all sources of randomness but conditioned on $\hat{G}$, and the other expectation is with respect to the remaining randomness (that is, $\hat{\boldsymbol{G}}$ ).

\section{ACKNOWLEDGEMENT}

The authors thank the reviewers for their constructive comments, which helped improve the quality of the paper.

\section{REFERENCES}

[1] T. L. Marzetta, "Noncooperative cellular wireless with unlimited numbers of base station antennas," IEEE Trans. Wireless Commun., vol. 9, no. 11 , pp. 3590-3600, Nov. 2010.

[2] "Delivery of broadcast content over LTE networks," https://tech.ebu.ch/docs/techreports/tr027.pdf.

[3] D. Lecompte and F. Gabin, "Evolved multimedia broadcast/multicast service (eMBMS) in LTE-advanced: overview and Rel-11 enhancements," IEEE Comm. Magazine, pp. 68-74, Nov. 2012.

[4] E. G. Larsson, "Joint beamforming and broadcasting in massive MIMO," in Proc. IEEE Workshop on Signal Processing Advances in Wireless Communications (SPAWC), 2015.
[5] M. Karlsson and E. G. Larsson, "On the operation of massive MIMO with and without transmitter CSI," in Proc. IEEE Workshop on Signal Processing Advances in Wireless Communications (SPAWC), 2014.

[6] X. Meng, X.-G. Xia, and X. Gao, "Constant-envelope omni-directional transmission with diversity in massive MIMO systems," in Proc. IEEE GLOBECOM, 2014.

[7] H. Yang, T. L. Marzetta, and A. Ashikhmin, "Multicast performance of large-scale antenna systems," in Proc. IEEE Workshop on Signal Processing Advances in Wireless Communications (SPAWC), June 2013, pp. 604-608.

[8] D. Christopoulos, S. Chatzinotas, and B. Ottersten, "Weighted fair multicast multigroup beamforming under per-antenna power constraints," IEEE Trans. Signal Process., vol. 62, pp. 5132-5142, Oct. 2014.

[9] — "Multicast multigroup beamforming for per-antenna power constrained large-scale arrays," in Proc. IEEE Workshop on Signal Processing Advances in Wireless Communications (SPAWC), 2015.

[10] H. Joudeh and B. Clerckx, "Sum rate maximization for MU-MISO with partial CSIT using joint multicasting and broadcasting," in Proc. IEEE Int. Conf. on Commun. (ICC), 2015.

[11] J. Zhu, R. Schober, and V. Bhargava, "Secure transmission in multicell massive MIMO systems," IEEE Trans. Wireless Comm., vol. 13, no. 9, pp. 4766-4781, 2014.

[12] _ "Linear precoding of data and artificial noise in secure massive MIMO systems," IEEE Trans. Wireless Commun., to appear.

[13] S. K. Mohammed and E. G. Larsson, "Per-antenna constant envelope precoding for large multi-user MIMO systems," IEEE Trans. Commun., vol. 61, pp. 1059-1071, Mar. 2013.

[14] C. Studer and E. G. Larsson, "PAR-aware large-scale multi-user MIMOOFDM downlink," IEEE J. Sel. Areas Commun., vol. 31, pp. 303-313, Feb. 2013.

[15] J. Pan and W.-K. Ma, "Constant envelope precoding for single-user large-scale MISO channels: Efficient precoding and optimal designs," IEEE J. Sel. Topics in Signal Proc., vol. 8, no. 5, pp. 982-995, Oct 2014.

[16] E. Björnson, E. G. Larsson, and M. Debbah, "Massive MIMO for maximal spectral efficiency: How many users and pilots should be allocated?" IEEE Trans. Wireless Commun., to appear.

[17] H. Yang and T. L. Marzetta, "Performance of conjugate and zeroforcing beamforming in large-scale antenna systems," IEEE J. Sel. Areas Commun., vol. 31, no. 2, pp. 172-179, Feb. 2013.

[18] H. Q. Ngo, E. G. Larsson, and T. L. Marzetta, "Energy and spectral efficiency of very large multiuser MIMO systems," IEEE Trans. Commun. vol. 61, pp. 1436-1449, Apr. 2013.

[19] _ , "Massive MU-MIMO downlink TDD systems with linear precoding and downlink pilots," in Proc. Annual Allerton Conf. on Commun., Cont., and Comp., Urbana-Champaign, Illinois, Oct. 2013, pp. 293-298.

[20] H. Q. Ngo and E. G. Larsson, "Blind estimation of effective downlink channel gains in massive MIMO," in Proc. IEEE Int. Conf. on Acoustics, Speech, and Signal Process. (ICASSP), Brisbane, Australia, Apr. 2015.

[21] G. Stewart, "The efficient generation of random orthogonal matrices with an application to condition estimators," SIAM Journal on Numerical Analysis, vol. 17, no. 3, pp. 403-409, 1980.

[22] X. Wu, W.-K. Ma, and A. M.-C. So, "Physical-layer multicasting by stochastic transmit beamforming and Alamouti space-time coding," IEEE Trans. Signal Process., pp. 4230-4245, Sep. 2013.

[23] B. Hassibi and B. M. Hochwald, "How much training is needed in multiple-antenna wireless links?" IEEE Trans. Inf. Theory, vol. 49, no. 4, pp. 951-963, Apr. 2003.

[24] H. Yang and T. L. Marzetta, "A macro-cellular wireless network with uniformly high user throughputs," in Proc. IEEE Vehicular Technology Conference, 2014.

[25] T. Söderström, Discrete-Time Stochastic Systems: Estimation and Control. Prentice-Hall, 1994.

[26] H. Shin and J. H. Lee, "Capacity of multiple-antenna fading channels: Spatial fading correlation, double scattering, and keyhole," IEEE Trans. Inf. Theory, vol. 49, pp. 2636-2647, Oct. 2003.

[27] A. M. Tulino and S. Verdu, Random Matrix Theory and Wireless Communications. Now Publishers, Foundations and Trends in Communications and Information Theory, 2004.

[28] H. Yang and T. L. Marzetta, "Capacity performance of multicell largescale antenna systems," in Proc. of Allerton Conference on Communication, Control, and Computing, 2013. 


\begin{tabular}{|c|c|c|c|c|c|c|c|c|}
\hline & $\begin{array}{l}\frac{\rho_{o}^{\mathrm{JBB}}}{\rho_{b}^{\mathrm{JBB}}} \\
{[\mathrm{dB}]}\end{array}$ & $\begin{array}{l}\rho_{d}^{\mathrm{JBB}} \\
{[\mathrm{dB}]}\end{array}$ & $\begin{array}{l}\rho_{b}^{\mathrm{JBB}} \\
{[\mathrm{dB}]}\end{array}$ & $\begin{array}{l}\rho_{o}^{\mathrm{JBB}} \\
{[\mathrm{dB}]}\end{array}$ & optimal $\epsilon$ & $\begin{array}{c}\frac{M^{\prime}-1}{M^{\prime}} \cdot \rho_{o} \beta_{o} \cdot \frac{\tau_{p}^{o} \rho_{o} \beta_{o}}{M^{\prime}+\tau_{p}^{o} \rho_{o} \beta_{o}}[\mathrm{~dB}] \\
\text { (power of useful signal } \\
\text { relative to } \\
\text { noise variance) }\end{array}$ & $\begin{array}{c}\rho_{o} \beta_{o} \cdot \frac{M^{\prime}}{M^{\prime}+\tau_{p}^{o} \rho_{o} \beta_{o}}[\mathrm{~dB}] \\
\text { (channel estimation } \\
\text { error relative to } \\
\text { noise variance) }\end{array}$ & $\begin{array}{c}\qquad \rho_{b}^{\prime} \beta_{o}[\mathrm{~dB}] \\
\text { (B-terminal interference } \\
\text { power relative to } \\
\text { noise variance) }\end{array}$ \\
\hline \begin{tabular}{ll|l} 
Figure & 3 & $\mathrm{JBB}^{\prime}$
\end{tabular} & 11.0 & 7.3 & -4.0 & 7.0 & N/A & 5.7 & -2.1 & -3.8 \\
\hline Figure 3 OA & 12.5 & 10.6 & -2.1 & 10.3 & 0.45 & 9.4 & -1.8 & $-\infty$ \\
\hline Figure $4 \mathrm{JBB}^{\prime}$ & 10.0 & 14.9 & 4.5 & 14.5 & N/A & 2.9 & -2.5 & -5.3 \\
\hline Figure 4 OA & 10.7 & 17.3 & 6.3 & 17.0 & 0.39 & 5.7 & -2.1 & $-\infty$ \\
\hline Figure $5 . \mathrm{JBB}^{\prime}$ & 7.4 & 10.8 & 2.7 & 10.1 & N/A & 9.1 & -1.8 & 2.9 \\
\hline Figure $5 . \mathrm{OA}$ & 9.6 & 13.7 & 3.7 & 13.2 & 0.41 & 12.4 & -1.7 & $-\infty$ \\
\hline Figure $6 \mathrm{JBB}^{\prime}$ & 5.8 & 0.9 & -6.0 & -0.2 & N/A & 9.3 & -1.8 & 4.6 \\
\hline Figure 6 OA & 8.3 & 3.5 & -5.4 & 2.9 & 0.37 & 12.4 & -1.7 & $-\infty$ \\
\hline
\end{tabular}

TABLE I

THE OPTIMAL VALUE $\epsilon$ FOR OA, AND THE STRENGTH OF THE NUMERATOR AND THE FIRST TWO TERMS IN THE DENOMINATOR OF 52, FOR RELEVANT OPERATING POINTS. 